\title{
Analytical solution of a two-dimensional model of liquid chromatography including moment analysis
}

\author{
Shamsul Qamar ${ }^{\mathrm{a}, \mathrm{b}, *}$, Farman U. Khan ${ }^{\mathrm{b}}$, Yasir Mehmood ${ }^{\mathrm{b}}$, Andreas Seidel-Morgenstern ${ }^{\mathrm{a}}$ \\ ${ }^{a}$ Max Planck Institute for Dynamics of Complex Technical Systems, \\ Sandtorstrasse 1, 39106 Magdeburg, Germany \\ ${ }^{b}$ Department of Mathematics, COMSATS Institute of Information Technology, \\ Park Road Chak Shahzad Islamabad, Pakistan
}

\begin{abstract}
This work presents an analysis of a two-dimensional model of a liquid chromatographic column. Constant flow rates and linear adsorption isotherms are assumed. Different sets of boundary conditions are considered, including injections through inner and outer regions of the column inlet cross section. The finite Hankel transform technique in combination with the Laplace transform method is applied to solve the model equations. The developed analytical solutions illustrate the influence and quantify the magnitude of the solute transport in radial direction. Comparing Dirichlet and Danckwerts boundary conditions, the predicted elution profiles differ significantly for large axial dispersion coefficients. For further analysis of the solute transport behavior, the temporal moments up to the fourth order are derived from the Laplace-transformed solutions. The analytical solutions for the concentration profiles and the moments are in good agreement with the numerical solutions of a high resolution flux limiting finite volume scheme. Results of different case studies
\end{abstract}

\footnotetext{
${ }^{*}$ Corresponding author. Tel: +49-391-6110454; fax: +49-391-6110500

Email addresses: qamar@mpi-magdeburg.mpg.de (Shamsul Qamar)
} 
are presented and discussed covering a wide range of mass transfer characteristics. The derived analytical solutions provide useful tools to quantify jointly occurring longitudinal and radial dispersion effects.

Key words: Liquid chromatography, two-dimensional model, finite Hankel and Laplace transformations, analytical solutions, moments analysis, dynamic simulation.

\section{Introduction}

Liquid chromatography applying cylindrical chromatographic columns is one of the most versatile separation techniques. It is widely used for analysis and purification in several important areas, e.g. in the pharmaceuticals, food, and fine chemical industries. The concept is successfully applied to realize difficult separation processes, as for instance the separation of enantiomers and the isolation of specific proteins from fermentation broths. In a chromatographic separation process a mobile phase percolates through a cylindrical column filled with fixed porous particles. This mobile phase carries the mixture components to be separated through the column. Components interacting more strongly with the particles will be transported slower compared to components with weaker interactions. Consequently, each component is characterized by an own concentration profile, which moves with a specific velocity. Provided the columns is long enough and transport processes do not destroy the separation, it is possible to collect at the outlet of the column in certain time periods pure fractions.

Different mathematical models with different degrees of complexity are available in the literature for describing the development of concentration profiles in chromatographic columns. 
Important and successful models are the general rate model (GRM), the lumped kinetic model (LKM) and the equilibrium dispersion model (EDM), see e.g. Carta (1988); Guiochon (2002); Guiochon and Lin (2003); Guiochon et al. (2006); Ruthven (1984). All these model need an important input information regarding the thermodynamic equilibrium of the distribution of the components between the mobile and stationary phases. They differ essentially regarding the consideration of unavoidable mass transfer processes, which cause undesired band broadening. Generally, only the most relevant concentration gradients occurring along the column axis are considered within the one-dimensional (1D) models. Gradients along the radial coordinate of the columns requiring the solution of two-dimensional (2D) models are typically neglected.

The models for chromatographic columns account for convection, adsorption, rate of exchange between the phases, intraparticle diffusion, film diffusion and dispersion. They consist of partial differential equations. Typically, solutions of the model equations can be only derived numerically due to the nonlinearity of the equilibrium functions.

A number of analytical solutions for one-, two- and three-dimensional advection-dispersion equations (ADEs) have been developed for predicting the transport of various contaminants in the subsurface. For example van Genuchten (1981) formulated several analytical solutions of the one-dimensional ADE subject to various initial and boundary conditions. Batu (1989, 1993) and Coimbra et al. (2003) presented analytical solutions of the twodimensional ADE with various source boundary conditions. Leij et al. (1991) and Park and Zhan (2001) derived analytical solutions for three-dimensional ADE. However, these models were mostly limited to ADE in Cartesian coordinates with steady uniform flow, see 
e.g. Park and Zhan (2001). Analytical solutions for two-dimensional ADE in cylindrical coordinates are particularly useful for analyzing problems of the two-dimensional solute transport in a porous medium system with steady uniform flow, see for example Chen et al. (2011a,b); Massab et al. (2011); Massabó et al. (2006); Park and Zhan (2001); Zhang et al. (2006).

Analytical solutions of models for column chromatography can be derived if linear adsorption isotherm can be assumed, see e.g. Carta (1988); Guiochon et al. (2006); Javeed et al. (2013); Li et al. (2003, 2004, 2011); Qamar et al. (2013). These solutions help tremendously to understand and analyze without extensive experiments the dynamics of concentration fronts moving through chromatographic columns. The availability of such solutions further provides useful tools to determine free transport parameters of the corresponding models. Finally, the solutions are most helpful for the validation of numerical methods needed to solve more general cases.

Provided analytical solutions of the column mass balances are available, condensed information in the form of moments of the outlet profiles can be easily obtained. Moment analysis has been comprehensively discussed in the literature, see for instance Antos (2003); Guiochon et al. (2006); Kubin (1964, 1965); Kucera (1965); Lenhoff (1987); Miyabe et al. (2000, 2003, 2007, 2009); Ruthven (1984); Schneider and Smith (1968); Suzuki (1973); Wolff et al. (1979, 1980). Recently, Javeed et al. (2013) and Qamar et al. (2013, 2014) used the Laplace transformation to derive analytical solutions of the equilibrium dispersive, the lumped kinetic and general rate models. Moreover, the authors also derived the moments of Laplace transformed solutions for different sets of boundary conditions (BCs). 
The Hankel transforms are the two-dimensional Fourier transforms of circularly symmetric functions, see for eaxmple Carslaw and Jaeger (1953); Sneddon (1972); Crank (1975). These are integral transformations whose kernels are Bessel functions and, thus, sometimes referred to as Bessel transforms. Solutions that are casted in term of Bessel functions arise frequently in boundary-value problems that involve radial and cylindrical coordinates. Therefore, Hankel transform is a practical technique for solving the boundary value problems expressed in cylindrical coordinates, allowing the radial coordinate to be eliminated. The purpose of this study is to extend the available knowledge by deriving analytical solutions for two-dimensional chromatographic models considering also the possibility of radial dispersion. An accordingly extended equilibrium dispersion model is applied subject to different boundary conditions. Specific injection profiles are assumed to amplify the effect of possible rate limitations of the mass transfer in the radial direction. Furthermore, both Dirichlet and Robin (Danckwerts) BCs are evaluated. The finite Hankel transform technique is applied to eliminate the radial coordinate. This technique provides a systematic, efficient and straightforward approach for obtaining analytical solutions for both transient and steady flow transport problems with a radial geometry. To derive exact analytical solutions of the model equations, the finite Hankel transform technique is coupled with the widely applied Laplace transform method, see for instance Carslaw and Jaeger (1953); Crank (1975); Chen et al. (2011a,b). The correctness of the derived solutions is proven in this paper by comparisons with numerical solutions generated with the high resolution flux-limiting finite volume scheme, see for example Javeed et al. (2011). Furthermore, temporal moments are derived as condensed information from the Laplace-transformed so- 
lutions, see e.g. Javeed et al. (2013); Qamar et al. (2013). Several case studies are carried out to illustrate the effect of the axial and radial dispersion coefficients on the effluent concentration profiles and the corresponding moments.

\section{Mathematical Model}

This study considers the transport of a solute in a two-dimensional chromatographic column of radial geometry as illustrated schematically in Figure 1. The injected solute migrates in the $z$-direction by advection and axial dispersion, whereas it spreads in the $r$-direction by radial dispersion. We neglect in this study flow rate variations and keep the interstitial velocity $u$ as constant. It is further assumed that the adsorption isotherm is linear with a Henry constant $a$. To trigger and amplify the effect of possible rate limitations of the mass transfer in the radial direction, the following specific injection conditions are assumed. By introducing a parameter $\tilde{r}$ the inlet cross section of the column is divided into an inner cylindrical core and an outer annular ring (see Figure 1). The injection profile is formulated in a general way allowing for injection either through an inner core, an outer ring or through the whole cross section. The latter case results if $\tilde{r}$ is set equal to the radius of the column denoted by $R$. Since in the latter case no initial radial gradients are provided, the solutions should converge into the solution of the simpler one-dimensional model which will be illustrated in the numerical test problems. It should be mentioned that the case of injecting the sample through the outer annular ring has some similarity with process of annular chromatography, see for example Thiele et al. (1973). However, during annular chromatography the column rotates. 
A two-dimensional equilibrium dispersive model of linear chromatography can be described by the following mass balance formulated in cylindrical coordinates

$$
\left(1+a \frac{1-\epsilon}{\epsilon}\right) \frac{\partial c}{\partial t}=-u \frac{\partial c}{\partial z}+D_{z} \frac{\partial^{2} c}{\partial z^{2}}+D_{r}\left(\frac{\partial^{2} c}{\partial r^{2}}+\frac{1}{r} \frac{\partial c}{\partial r}\right)
$$

Here, $c(r, z, t)$ denotes concentration of the solute, $t$ is time, and $D_{z}$ and $D_{r}$ represent the longitudinal and radial dispersion coefficients, respectively. Moreover, $\epsilon$ is the external porosity following $\epsilon \in(0,1)$.

To simplify the analysis, let us define some dimensionless variables

$$
x=\frac{z}{L}, \quad \rho=\frac{r}{R}, \quad \tau=\frac{t}{\frac{L}{u}\left(1+a \frac{1-\epsilon}{\epsilon}\right)}, \quad P e_{z}=\frac{L u}{D_{z}}, \quad P e_{r}=\frac{R^{2} u}{D_{r} L}
$$

where $L$ denotes length of the column. Inserting these variables into Eq. (1) yields

$$
\frac{\partial c}{\partial \tau}=-\frac{\partial c}{\partial x}+\frac{1}{P e_{z}} \frac{\partial^{2} c}{\partial x^{2}}+\frac{1}{P e_{r}}\left(\frac{\partial^{2} c}{\partial \rho^{2}}+\frac{1}{\rho} \frac{\partial c}{\partial \rho}\right)
$$

The initial condition for a uniformly pre-equilibrated column are given as

$$
c(\rho, x, \tau=0)=c_{\text {init }}, \quad 0 \leq x \leq 1, \quad 0<\rho \leq 1
$$

where $c_{\text {init }}$ is the initial concentration of the solute in the column. The above equation is also subjected to the boundary conditions. The following boundary conditions at $\rho=0$ and $\rho=1$ are assumed

$$
\frac{\partial c(\rho=0, x, \tau)}{\partial \rho}=0, \quad \frac{\partial c(\rho=1, x, \tau)}{\partial \rho}=0
$$

The first boundary condition corresponds to the symmetry of radial profile, while the second condition represents the impermeability of the column wall. Moreover, two sets of 
boundary conditions are considered at the column inlet and outlet which are summarized below.

Case 1: Concentration pulse of finite width is injected as Dirichlet inlet BCs:

For injection in the inner circular region, it is expressed as

$$
c(\rho, x=0, \tau)= \begin{cases}c_{\mathrm{inj}}, & \text { if } 0 \leq \rho \leq \tilde{\rho} \text { and } 0 \leq \tau \leq \tau_{\mathrm{inj}} \\ 0, & \text { if } \tilde{\rho}<\rho \leq 1 \text { or } \tau>\tau_{\text {inj }}\end{cases}
$$

while, for injection in the outer annular zone, it is described as

$$
c(\rho, x=0, \tau)= \begin{cases}c_{\mathrm{inj}}, & \text { if } \tilde{\rho}<\rho \leq 1 \text { and } 0 \leq \tau \leq \tau_{\mathrm{inj}} \\ 0, & \text { if } 0 \leq \rho \leq \tilde{\rho} \text { or } \tau>\tau_{\text {inj }}\end{cases}
$$

The symbol $c_{\text {inj }}$ represents the concentration of injected solute solution and

$$
\tilde{\rho}=\tilde{r} / R
$$

For injection over the whole inlet cross section of the column, either $\tilde{\rho}=1$ in Eq. (6) or $\tilde{\rho}=0$ in Eq. (7).

A Neumann boundary condition is considered at the outlet for a column of hypothetically infinite length, $x=\infty$,

$$
\left.\frac{\partial c}{\partial x}\right|_{x=\infty}=0 .
$$

Case 2: Concentration pulse of finite width injected as Danckwerts inlet BCs :

For the inner zone injection, this boundary condition is expressed as

$$
c(\rho, x=0, \tau)-\frac{1}{P e_{z}} \frac{\partial c(\rho, x=0, \tau)}{\partial x}= \begin{cases}c_{\text {inj }}, & \text { if } 0 \leq \rho \leq \tilde{\rho} \text { and } 0 \leq \tau \leq \tau_{\text {inj }}, \\ 0, & \tilde{\rho}<\rho \leq 1 \text { or } \tau>\tau_{\text {inj }},\end{cases}
$$


while, for the injection through outer annular zone it is given as

$$
c(\rho, x=0, \tau)-\frac{1}{P e_{z}} \frac{\partial c(\rho, x=0, \tau)}{\partial x}=\left\{\begin{array}{cl}
c_{\text {inj }}, & \text { if } \tilde{\rho}<\rho \leq 1 \text { and } 0 \leq \tau \leq \tau_{\text {inj }}, \\
0, & 0 \leq \rho \leq \tilde{\rho} \text { or } \tau>\tau_{\text {inj }},
\end{array}\right.
$$

together with the Neumann condition at the outlet of a finite length column

$$
\left.\frac{\partial c}{\partial x}\right|_{x=1}=0
$$

\section{Derivation of analytical solution}

The chromatographic model in Eq. (3) and its associated initial and boundary conditions are analytically solved by successive implementation of the finite Hankel transform and the Laplace transform, see for example Chen et al. (2011a,b). The Hankel Transformation of Eq. (3) with respect to $\rho$ gives

$$
\frac{\partial c_{H}}{\partial \tau}=-\frac{\partial c_{H}}{\partial x}+\frac{1}{P e_{z}} \frac{\partial^{2} c_{H}}{\partial x^{2}}-\frac{1}{P e_{r}} \lambda_{n}^{2} c_{H}
$$

where $\lambda_{n}$ is the finite Hankel transform parameter as determined by the transcendental equation $\frac{d J_{0}\left(\lambda_{n}\right)}{d \rho}=-J_{1}\left(\lambda_{n}\right)=0$. Here, $J_{0}($.$) and J_{1}($.$) are the zeroth and first order Bessel$ functions of the first kind and $c_{H}\left(\lambda_{n}, x, \tau\right)$ is the the zeroth-order finite Hankel transform of $c(\rho, x, \tau)$ as defined below (c.f. Carslaw and Jaeger (1953); Sneddon (1972); Crank (1975);

Chen et al. (2011a,b))

$$
c_{H}\left(\lambda_{n}, x, \tau\right)=H[c(\rho, x, \tau)]=\int_{0}^{1} c(\rho, x, \tau) J_{0}\left(\lambda_{n} \rho\right) \rho d \rho .
$$

The inverse Hankel transform is given as

$$
c(\rho, x, \tau)=2 c_{H}\left(\lambda_{n}=0, x, \tau\right)+2 \sum_{n=1}^{\infty} c_{H}\left(\lambda_{n}, x, \tau\right) \frac{J_{0}\left(\lambda_{n} \rho\right)}{\left|J_{0}\left(\lambda_{n}\right)\right|^{2}} .
$$


Accordingly, the initial condition in Eq. (4) after taking the Hankel transform becomes

$$
c_{H}\left(\lambda_{n}, x, \tau=0\right)=c_{\text {init }} F\left(\lambda_{n}\right) .
$$

For injection at the inner cylindrical core, $F\left(\lambda_{n}\right)$ is given as

$$
F\left(\lambda_{n}\right)= \begin{cases}\frac{\tilde{\rho}^{2}}{2}, & \text { if } \lambda_{n}=0, \\ \frac{\tilde{\rho}}{\lambda_{n}} J_{1}\left(\lambda_{n} \tilde{\rho}\right), & \text { if } \lambda_{n} \neq 0,\end{cases}
$$

while for injection at the outer annular ring, it becomes

$$
F\left(\lambda_{n}\right)= \begin{cases}\left(\frac{1}{2}-\frac{\tilde{\rho}^{2}}{2}\right), & \text { if } \lambda_{n}=0, \\ -\frac{\tilde{\rho}}{\lambda_{n}} J_{1}\left(\lambda_{n} \tilde{\rho}\right), & \text { if } \lambda_{n} \neq 0 .\end{cases}
$$

Here, $\tilde{\rho}$ is given by Eq. (8).

By applying the Laplace transformation on Eq. (13) with respect to $\tau$, we get

$$
\frac{1}{P e_{z}} \frac{\partial^{2} \bar{c}_{H}}{\partial x^{2}}-\frac{\partial \bar{c}_{H}}{\partial x}-\left(\frac{1}{P e_{r}} \lambda_{n}^{2}+s\right) \bar{c}_{H}=-c_{\text {init }} F\left(\lambda_{n}\right) .
$$

The general solution of this equation is given as

$$
\bar{c}_{H}=A_{0} e^{m_{1} x}+B_{0} e^{m_{2} x}+\frac{c_{\mathrm{init}} F\left(\lambda_{n}\right)}{s+\frac{1}{P e_{r}} \lambda_{n}^{2}},
$$

where

$$
m_{1,2}=\frac{P e_{z}}{2} \pm \sqrt{\left(\frac{P e_{z}}{2}\right)^{2}+\frac{P e_{z}}{P e_{r}} \lambda_{n}^{2}+P e_{z} s},
$$

and $A_{0}$ and $B_{0}$ are constant to be determined from the given boundary conditions. In Eq. (21), the plus sign (upper case) is selected for calculating $m_{1}$ and the minus sign (lower case) is considered for calculating $m_{2}$.

Case 1: Concentration pulse of finite width is injected as Dirichlet inlet BCs: 
The Hankel transformations of Eqs. (6) (or Eqs. (7)) and (9) are given as

$$
\begin{gathered}
c_{H}\left(\lambda_{n}, x=0, t\right)= \begin{cases}c_{\mathrm{inj}} F\left(\lambda_{n}\right), & \text { if } 0 \leq \tau \leq \tau_{\mathrm{inj}}, \\
0, & \text { if } \tau>\tau_{\mathrm{inj}},\end{cases} \\
\left.\frac{\partial c_{H}\left(\lambda_{n}, x, \tau\right)}{\partial x}\right|_{x=\infty}=0 .
\end{gathered}
$$

Here, $F\left(\lambda_{n}\right)$ is given by Eq. (17) for inner injection and by Eq. (18) for outer annular injection.

After applying the Laplace transformation on boundary conditions in Eqs. (22) and (23), we obtain

$$
\bar{c}_{H}\left(\lambda_{n}, x=0, s\right)=\frac{c_{\mathrm{inj}} F\left(\lambda_{n}\right)}{s}\left(1-e^{-s \tau_{\mathrm{inj}}}\right),\left.\quad \frac{\partial \bar{c}_{H}}{\partial x}\right|_{x=\infty}=0 .
$$

Now using Eq. (24) in Eq. (20), we obtain

$$
A_{0}=0, \quad B_{o}=\left(\frac{c_{\mathrm{inj}}\left(1-e^{-s \tau_{\mathrm{inj}}}\right)}{s}-\frac{c_{\mathrm{init}}}{s+\frac{1}{P e_{r}} \lambda_{n}^{2}}\right) F\left(\lambda_{n}\right)
$$

Thus, the solution in Eq. (20) takes the form

$$
\bar{c}_{H}\left(\lambda_{n}, x, s\right)=\left(\frac{c_{\mathrm{inj}}\left(1-e^{-s \tau_{\mathrm{inj}}}\right)}{s}-\frac{c_{\mathrm{init}}}{s+\frac{1}{P e_{r}} \lambda_{n}^{2}}\right) F\left(\lambda_{n}\right) e^{m_{2} x}+\frac{c_{\mathrm{init}} F\left(\lambda_{n}\right)}{s+\frac{1}{P e_{r}} \lambda_{n}^{2}}
$$

where $m_{2}$ is given by Eq. (21) for lower negative sign.

Using the inverse Laplace transformation on Eq. (26), the solution in actual time domain is given as (c.f. van Genuchten (1981))

$$
c_{H}\left(\lambda_{n}, x, \tau\right)=\left\{\begin{array}{l}
A\left(\lambda_{n}, x, \tau\right), \quad \text { if } 0 \leq \tau \leq \tau_{\text {inj }}, \\
A\left(\lambda_{n}, x, \tau\right)-A\left(\lambda_{n}, x, \tau-\tau_{\text {inj }}\right), \quad \text { if } \tau>\tau_{\text {inj }} .
\end{array}\right.
$$


Letting

$$
v=\sqrt{\left(P e_{z}\right)^{2}+4 \frac{P e_{z}}{P e_{r}} \lambda_{n}^{2}},
$$

then

$$
\begin{aligned}
A\left(\lambda_{n}, x, \tau\right)= & \frac{c_{\mathrm{inj}} F\left(\lambda_{n}\right)}{2}\left[e^{\frac{\left(P e_{z}-v\right) x}{2}} \operatorname{erfc}\left(\frac{P e_{z} x-v \tau}{2 \sqrt{P e_{z} \tau}}\right)+e^{\frac{\left(P e_{z}+v\right) x}{2}} \operatorname{erfc}\left(\frac{P e_{z} x+v \tau}{2 \sqrt{P e_{z} \tau}}\right)\right] \\
& -\frac{c_{\text {init }} F\left(\lambda_{n}\right) e^{-\frac{\lambda_{n}^{2} \tau}{P e_{r}}}}{2}\left[\operatorname{erfc}\left(\frac{x-\tau}{2 \sqrt{\tau / P e_{z}}}\right)+e^{P e_{z} x} \operatorname{erfc}\left(\frac{x+\tau}{2 \sqrt{\tau / P e_{z}}}\right)-2\right] .
\end{aligned}
$$

Using Eq. (15), the final solution is

$$
c(\rho, x, \tau)=\left\{\begin{array}{l}
2 A\left(\lambda_{n}=0, x, \tau\right)+2 \sum_{n=1}^{\infty} A\left(\lambda_{n}, x, \tau\right) \frac{J_{0}\left(\lambda_{n} \rho\right)}{\left|J_{0}\left(\lambda_{n}\right)\right|^{2}}, \quad \text { if } 0 \leq \tau \leq \tau_{\text {inj }}, \\
2\left[A\left(\lambda_{n}=0, x, \tau\right)-A\left(\lambda_{n}=0, x, \tau-\tau_{\text {inj }}\right)\right] \\
+2 \sum_{n=1}^{\infty}\left[A\left(\lambda_{n}, x, \tau\right)-A\left(\lambda_{n}, x, \tau-\tau_{\text {inj }}\right)\right] \frac{J_{0}\left(\lambda_{n} \rho\right)}{\left|J_{0}\left(\lambda_{n}\right)\right|^{2}}, \quad \text { if } \quad \tau>\tau_{\text {inj }} .
\end{array}\right.
$$

Case 2: Concentration pulse of finite width injected as Danckwerts inlet BCs :

The Hankel transformations of Eqs. (10) (or Eqs. (11)) and (12) are given as

$$
c_{H}\left(\lambda_{n}, x=0, \tau\right)-\frac{1}{P e_{z}} \frac{\partial c_{H}\left(\lambda_{n}, x=0, \tau\right)}{\partial x}= \begin{cases}c_{\mathrm{inj}} F\left(\lambda_{n}\right), & \text { if } 0 \leq \tau \leq \tau_{\mathrm{inj}} \\ 0, & \text { if } \tau>\tau_{\mathrm{inj}},\end{cases}
$$

together with the Neumann condition at the outlet of the column

$$
\left.\frac{\partial c_{H}\left(\lambda_{n}, x, \tau\right)}{\partial x}\right|_{x=1}=0 .
$$

Once again, $F\left(\lambda_{n}\right)$ is given by Eq. (17) for the inner injection and by Eq. (18) for the outer annular injection.

After applying the Laplace transformation on these boundary conditions, we get

$$
\bar{c}_{H}\left(\lambda_{n}, x=0, s\right)-\frac{1}{P e_{z}} \frac{\partial \bar{c}_{H}\left(\lambda_{n}, x=0, s\right)}{\partial x}=\frac{c_{\mathrm{inj}} F\left(\lambda_{n}\right)}{s}\left(1-e^{-s \tau_{\mathrm{inj}}}\right),
$$


and

$$
\left.\frac{\partial \bar{c}_{H}}{\partial x}\right|_{x=1}=0 .
$$

Now using Eqs. (33) and (34) in Eq. (20), we obtain

$$
A_{0}=\frac{m_{2} e^{m_{2}}\left(\frac{c_{\mathrm{inj}} F\left(\lambda_{n}\right)}{s}\left(1-e^{-s \tau_{\mathrm{inj}}}\right)-\frac{c_{\mathrm{init}} F\left(\lambda_{n}\right)}{s+\frac{1}{P e_{r}} \lambda_{n}^{2}}\right)}{m_{2} e^{m_{2}}\left(1-\frac{m_{1}}{P e_{z}}\right)-m_{1} e^{m_{1}}\left(1-\frac{m_{2}}{P e_{z}}\right)}
$$

and

$$
B_{0}=-\frac{m_{1} e^{m_{1}}\left(\frac{c_{\mathrm{inj}} F\left(\lambda_{n}\right)}{s}\left(1-e^{-s \tau_{\mathrm{inj}}}\right)-\frac{c_{\mathrm{init}} F\left(\lambda_{n}\right)}{s+\frac{1}{P e_{r}} \lambda_{n}^{2}}\right)}{m_{2} e^{m_{2}}\left(1-\frac{m_{1}}{P e_{z}}\right)-m_{1} e^{m_{1}}\left(1-\frac{m_{2}}{P e_{z}}\right)}
$$

Thus, the solution in Eq. (20) takes the form

$$
\bar{c}_{H}\left(\lambda_{n}, x, s\right)=\frac{F\left(\lambda_{n}\right)\left[m_{2} e^{m 2+m_{1} x}-m_{1} e^{m 1+m_{2} x}\right]\left[\frac{c_{\mathrm{inj}}}{s}\left(1-e^{-s \tau_{\mathrm{inj}}}\right)-\frac{c_{\text {init }}}{s+\frac{1}{P e_{r}} \lambda_{n}^{2}}\right]}{m_{2} e^{m_{2}}\left(1-\frac{m_{1}}{P e_{z}}\right)-m_{1} e^{m_{1}}\left(1-\frac{m_{2}}{P e_{z}}\right)}+\frac{c_{\text {init }} F\left(\lambda_{n}\right)}{s+\frac{1}{P e_{r}} \lambda_{n}^{2}} .
$$

Using the inverse Laplace transformation on Eq. (37), the solution in actual time domain is given as (c.f. Chen et al. (2011b); van Genuchten (1981))

$$
c_{H}\left(\lambda_{n}, x, \tau\right)=\left\{\begin{array}{l}
B\left(\lambda_{n}, x, \tau\right), \quad \text { if } 0 \leq \tau \leq \tau_{\text {inj }}, \\
B\left(\lambda_{n}, x, \tau\right)-B\left(\lambda_{n}, x, \tau-\tau_{\text {inj }}\right), \quad \text { if } \tau>\tau_{\text {inj }},
\end{array}\right.
$$

where

$$
\begin{aligned}
B\left(\lambda_{n}, x, \tau\right)= & c_{\text {inj }} F\left(\lambda_{n}\right)\left[1-\sum_{m=1}^{\infty} \varphi\left(\vartheta_{m}, x\right) \cdot \psi\left(\lambda_{n}, \vartheta_{m}, x, \tau\right)\right] \\
& +c_{\text {init }} F\left(\lambda_{n}\right) e^{-\frac{\lambda_{n}^{2} \tau}{P e_{r}}}\left[\sum_{m=1}^{\infty} \varphi\left(\vartheta_{m}, x\right) \cdot \psi\left(\lambda_{n}=0, \vartheta_{m}, x, \tau\right)\right]
\end{aligned}
$$


Moreover

$$
\begin{gathered}
\varphi\left(\vartheta_{m}, x\right)=\frac{2 P e_{z} \vartheta_{m}\left[\frac{P e_{z}}{2} \sin \left(\vartheta_{m} x\right)+\vartheta_{m} \cos \left(\vartheta_{m} x\right)\right]}{\left(\frac{P e_{z}^{2}}{4}+P e_{z}+\vartheta_{m}^{2}\right)\left(\frac{P e_{z}^{2}}{4}+\vartheta_{m}^{2}\right)}, \\
\psi\left(\lambda_{n}, \vartheta_{m}, x, \tau\right)=\frac{\frac{P e_{z}}{P e_{r}} \lambda_{n}^{2} e^{\frac{P e_{z}}{2} x}+\left(\vartheta_{m}^{2}+\frac{P e_{L}^{2}}{4}\right) \exp \left(\frac{P e_{z}}{2} x-\frac{P e_{z}}{4} \tau-\frac{\lambda_{n}^{2}}{P e_{r}} \tau-\frac{\vartheta_{m}^{2}}{P e_{z}} \tau\right)}{\vartheta_{m}^{2}+\frac{P e_{L}^{2}}{4}+\frac{P e_{z}}{P e_{r}} \lambda_{n}^{2}},
\end{gathered}
$$

and the eigenvalues $\vartheta_{m}$ are the positive roots of the following equation

$$
P e_{z} \cot \left(\vartheta_{m}\right)=\frac{\vartheta_{m}^{2}-\frac{P e_{z}^{2}}{4}}{\vartheta_{m}}
$$

Then final solution is given as (c.f. Eq. (15))

$$
c(\rho, x, \tau)=\left\{\begin{array}{l}
2 B\left(\lambda_{n}=0, x, \tau\right)+2 \sum_{n=1}^{\infty} B\left(\lambda_{n}, x, \tau\right) \frac{J_{0}\left(\lambda_{n} \rho\right)}{\left|J_{0}\left(\lambda_{n}\right)\right|^{2}}, \quad \text { if } 0 \leq \tau \leq \tau_{\text {inj }}, \\
2\left[B\left(\lambda_{n}=0, x, \tau\right)-B\left(\lambda_{n}=0, x, \tau-\tau_{\text {inj }}\right)\right] \\
+2 \sum_{n=1}^{\infty}\left[B\left(\lambda_{n}, x, \tau\right)-B\left(\lambda_{n}, x, \tau-\tau_{\text {inj }}\right)\right] \frac{J_{0}\left(\lambda_{n} \rho\right)}{\left|J_{0}\left(\lambda_{n}\right)\right|^{2}}, \quad \text { if } \quad \tau>\tau_{\text {inj }} .
\end{array}\right.
$$

\section{Moment Analysis}

Moment analysis is known to be an effective method for deducing important information about the retention equilibrium and mass transfer kinetics in a chromatographic column. A set of statistical temporal moments can define the appearance of the plotted elution profile. For example, the appropriate forms of the first, second, third and fourth moments will describe the mean, spread, skew, and kurtosis, respectively, of the distribution. The experiential values measured for these moments can be compared with their theoretical expressions to estimate dispersion and other mass transfer coefficients.

Normalized averaged $i$-th moments of the band profile at any position in the column can 
be obtained through the following expression

$$
\mu_{i, \mathrm{av}}=\frac{\int_{0}^{\infty} c_{\mathrm{av}}(x, \tau) \tau^{i} d \tau}{\mu_{0, \mathrm{av}}}, \quad i=1,2,3, \cdots
$$

where

$$
c_{\mathrm{av}}(x, \tau)=2 \int_{0}^{1} c(\rho, x, \tau) \rho d \rho,
$$

and for the the zeroth moment (mass balance) holds

$$
\mu_{0, \mathrm{av}}=\int_{0}^{\infty} c_{\mathrm{av}}(x, \tau) d \tau
$$

Due to mass balance considerations for this zeroth moment holds

$$
\mu_{0, \mathrm{av}}=c_{\mathrm{inj}, \mathrm{av}} \tau_{\mathrm{inj}}
$$

where $c_{\mathrm{inj}, \mathrm{av}}=c_{\mathrm{inj}} \tilde{\rho}^{2}$ for the inner circular zone injection and $c_{\mathrm{inj}, \mathrm{av}}=c_{\mathrm{inj}}\left(1-\tilde{\rho}^{2}\right)$ for the outer annular zone injection. Moreover, according to Eq. (2), $\tau_{\text {inj }}=\frac{t_{\text {inj }}}{\frac{L}{u}\left(1+a \frac{1-\epsilon}{\epsilon}\right)}$.

When mass transfer in the radial direction is assumed hypothetically to be infinitely fast, $c_{\mathrm{av}}(x, \tau)=c(\rho, x, \tau)$, that corresponds to the 1D case presented in Qamar et al. (2013).

Due to its moment generating properties the Laplace transformation can be used as a basic tool to derive analytical expressions for the moments. In this study, analytical temporal moments are derived as functions of radial coordinate $\rho$ at the outlet of the column $(x=1)$ considering $c_{\text {init }}=0$. Afterwards, these moments are used to obtain the aforementioned averaged moments by integrating over $\rho$. The following property of the Laplace transform was used to determine the analytical moments from the Laplace and Hankel transformed concentration $\bar{c}_{H}$ in Eq. (26) or Eq. (37):

$$
\mu_{i, H}=(-1)^{i} \lim _{s \rightarrow 0} \frac{d^{i}\left(\bar{c}_{H}\left(\lambda_{n}, x, s\right)\right)}{d s^{i}}, \quad i=0,1,2, \cdots
$$


The actual moments $\mu_{i}(\rho)$ are generated from Eq. (15) by taking moments of the concentrations on both sides of that relation. On multiplying both sides of Eq. (15) with $\tau^{i}$ and integrating over tau from 0 to $\infty$, we obtain

$$
\mu_{i}(\rho)=2 \mu_{i, H}\left(\lambda_{n}=0\right)+2 \sum_{n=1}^{\infty} \mu_{i, H}\left(\lambda_{n}\right) \frac{J_{0}\left(\lambda_{n} \rho\right)}{\left|J_{0}\left(\lambda_{n}\right)\right|^{2}}
$$

From the above moments, the averaged non-normalized temporal moments $M_{i, \text { av }}$ can be calculated as

$$
M_{i, \mathrm{av}}=2 \int_{0}^{1} \mu_{i}(\rho) \rho d \rho, \quad i=0,1,2, \cdots
$$

Finally, the normalized averaged temporal moments (c.f. Eq. (44)) which are widely used in chemical engineering (Guiochon et al. (2006)) are available as

$$
\mu_{i, \mathrm{av}}=\frac{M_{i, \mathrm{av}}}{\mu_{0, \mathrm{av}}}, \quad \mu_{0, \mathrm{av}}=M_{0, \mathrm{av}}, \quad i=1,2,3, \cdots
$$

For evaluation of the solute transport behavior, the above averaged temporal moments $\mu_{i, \text { av }}$ up to the fourth order are derived. These moments can be used to get finally also the first three averaged central moments defined below (e.g. Guiochon et al. (2006)):

$$
\begin{aligned}
& \mu_{2, \mathrm{av}}^{\prime}=\mu_{2, \mathrm{av}}-\mu_{1, \mathrm{av}}^{2} . \\
& \mu_{3, \mathrm{av}}^{\prime}=\mu_{3, \mathrm{av}}-3 \mu_{1, \mathrm{av}} \mu_{2, \mathrm{av}}+2 \mu_{1, \mathrm{av}}^{3} . \\
& \mu_{4, \mathrm{av}}^{\prime}=\mu_{4, \mathrm{av}}-4 \mu_{1, \mathrm{av}} \mu_{3, \mathrm{av}}+6 \mu_{1, \mathrm{av}}^{2} \mu_{2, \mathrm{av}}-3 \mu_{1, \mathrm{av}}^{4} .
\end{aligned}
$$

The corresponding $i$-th central moments of the band profile at the outlet of a column of length $x=1$ are numerically obtained using the expression

$$
\mu_{i, \mathrm{av}}^{\prime}=\frac{\int_{0}^{\infty} c_{\mathrm{av}}(x=1, \tau)\left(\tau-\mu_{1}\right)^{i} d \tau}{\mu_{0, \mathrm{av}}}, \quad i=2,3,4, \cdots
$$


where, $\mu_{0, \text { av }}$ for $x=1$ is given by Eq. (46). The trapezoidal rule is applied to numerically approximate the integrals in Eqs. (44)-(46) and (55). The central moments are well known in chemical engineering due to their physical meanings, see e.g. Guiochon et al. (2006). Hereby the zeroth moment corresponds to mass balance (peak areas), the first to mean retention times, the second to variance around the mean resentence time, the third to peak asymmetry (skewness) and the forth to kurtosis. The values of these moments should be in agreement with those provided by Qamar et al. (2013) based on an analysis of the 1D case, in which $D_{z}$ has an effect on their values.

Case 1: Concentration pulse of finite width injected as Dirichlet inlet BCs (Eq. (22) and (23)).

The moments of $\bar{c}_{H}$ in Eq. (26) are obtained through Eq. (48) which are summarized below. The zeroth moment: It is obtained from Eqs. (26) and (48) for $i=0$ :

$$
\mu_{0, H}=\tau_{\mathrm{inj}} c_{\mathrm{inj}} F\left(\lambda_{n}\right) \exp \left(\frac{P e_{z}-v}{2}\right),
$$

where $v$ is given by Eq. (28). Moreover, $F\left(\lambda_{n}\right)$ is given by Eq. (17) for inner zone injection and by Eq. (18) for outer annular zone injection.

First moment: It is calculated from Eqs. (26) and (48) for $i=1$ :

$$
\mu_{1, H}=\left[\frac{\tau_{\text {inj }}}{2}+\frac{P e_{z}}{v}\right] \mu_{0, H}
$$

Second moment: It is derived from Eqs. (26) and (48) for $i=2$ :

$$
\mu_{2, H}=\left[\frac{\tau_{\mathrm{inj}}^{2}}{3}+\frac{\tau_{\mathrm{inj}} P e_{z}}{v}+\frac{2 P e_{z}^{2}+P e_{z}^{2} v}{v^{3}}\right] \mu_{0, H}
$$


Third moment: The third temporal moment based on Eqs. (26) and (48) for $i=3$ :

$$
\mu_{3, H}=\left[\frac{\tau_{\mathrm{inj}}^{3}}{4}+\frac{\tau_{\mathrm{inj}}^{2} P e_{z}}{v}+\frac{3 \tau_{\mathrm{inj}}}{2} \frac{\left(2 P e_{z}^{4}+P e_{z}^{2} v^{3}\right)}{v^{5}}+\frac{12 P e_{z}^{3}+P e_{z}^{3} v^{2}+6 P e_{z}^{3} v}{v^{5}}\right] \mu_{0, H}
$$

Fourth moment: The fourth moment is obtained as (c.f. Eq. (48) for $i=4$ )

$$
\begin{aligned}
\mu_{4, H}= & {\left[\frac{\tau_{\text {inj }}^{4}}{5}+\frac{P e_{z} \tau_{\text {inj }}^{3}}{v}+\frac{2 \tau_{\text {inj }}^{2}\left(2 P e_{z}^{2}+P e_{z}^{2} v\right)}{v^{3}}+\frac{2 \tau_{\text {inj }}\left(12 P e_{z}^{3}+P e_{z}^{3} v^{2}+6 P e_{z}^{3} v\right)}{v^{5}}\right.} \\
& \left.+\frac{P e_{L}^{4}\left[120+12 v^{2}+\left(v^{2}+60\right) v\right]}{v^{7}}\right] \mu_{0, H} .
\end{aligned}
$$

The above moments correctly reduce to the $1 \mathrm{D}$ case for $\lambda_{n}=0$. In that case $F\left(\lambda_{n}=0\right)=1$.

\section{Case 2: Concentration pulse of finite width injected as Danckwerts inlet BCs}

(Eqs. (10) and (12)).

Here, the temporal moments of concentration profile given by Eq. (37) are calculated.

The zeroth moment: The zeroth moment is calculated by using Eqs. (37) and (48). Let us define

$$
\alpha=\sqrt{1+\frac{4 \lambda_{n}^{2}}{P e_{z} P e_{r}}}
$$

Then, it is given as

$$
\mu_{0, H}=\frac{4 \tau_{\mathrm{inj}} c_{\mathrm{inj}} \alpha F\left(\lambda_{n}\right) e^{P e_{z}}}{(\alpha+1)^{2} e^{\frac{P e_{z}(\alpha+1)}{2}}-(\alpha-1)^{2} e^{-\frac{P e_{z}(\alpha-1)}{2}}} .
$$

\section{First moment:}

The first temporal moment is calculated from Eqs. (37) and (48) for $i=1$. Let us define

$$
\alpha_{1}=-4+P e_{z} \alpha^{4} \tau_{\text {inj }}+\left(4+\left(\tau_{\text {inj }}+4\right) P e_{z}\right) \alpha^{2}, \quad \alpha_{2}=2 P e_{z}\left(1+\tau_{\text {inj }}\right) \alpha^{3}+2 P e_{z} \alpha
$$


Then, it comes out to be

$$
\mu_{1, H}=\frac{1}{2 \alpha^{2} P e_{z}}\left[\frac{\left(\alpha_{1}+\alpha_{2}\right) e^{\frac{P e_{z}(\alpha+1)}{2}}-\left(\alpha_{1}-\alpha_{2}\right) e^{-\frac{P e_{z}(\alpha-1)}{2}}}{(\alpha+1)^{2} e^{\frac{P e_{z}(\alpha+1)}{2}}-(\alpha-1)^{2} e^{-\frac{P e_{z}(\alpha-1)}{2}}}\right] \mu_{0, H} .
$$

Second moment: Let us define

$$
\begin{aligned}
& \alpha_{3}=\tau_{\mathrm{inj}}^{2} \alpha^{5} P e_{z}^{2}+P e_{z}\left[\left(3+6 \tau_{\mathrm{inj}}+\tau_{\mathrm{inj}}^{2}\right) P e_{z}+6 \tau_{\mathrm{inj}}\right] \alpha^{3}+\left(3 P e_{z}^{2}+24-6 \tau_{\mathrm{inj}} P e_{z}\right) \alpha \\
& \alpha_{4}=-2 \tau_{\mathrm{inj}}\left(\tau_{\mathrm{inj}}+\frac{3}{2}\right) P e_{z}^{2} \alpha^{4}-\left[\left(3 \tau_{\mathrm{inj}}+6\right) P e_{z}^{2}+12 P e_{z}\right] \alpha^{2}+48+12 P e_{z} \\
& \alpha_{5}=\alpha^{2} P e_{z}-2, \quad \alpha_{6}=\left(P e_{z}+2\right) \alpha, \quad \alpha_{7}=12 \alpha^{5}-12 \alpha \\
& \alpha_{8}=\left\{\tau_{\mathrm{inj}}^{2} \alpha^{6} P e_{z}^{2}+\left[\left(-9-2 \tau_{\mathrm{inj}}^{2}\right) P e_{z}^{2}+6 \tau_{\mathrm{inj}} P e_{z}\right] \alpha^{4}+\left[24+\left(\tau_{\mathrm{inj}}^{2}+18\right) P e_{z}^{2}+72 P e_{z}\right] \alpha^{2}\right. \\
& \left.-72-9 P e_{z}^{2}+\left(-72-6 \tau_{\mathrm{inj}}\right) P e_{z}\right\} \alpha^{3} . \\
& \alpha_{9}=\frac{1}{3 \alpha^{6} P e_{z}^{2}\left[(\alpha+1)^{2} e^{\frac{P e_{z}(\alpha+1)}{2}}-(\alpha-1)^{2} e^{-\frac{P e_{z}(\alpha-1)}{2}}\right]^{2}}
\end{aligned}
$$

Then, it is expressed as

$$
\begin{aligned}
\mu_{2, H}= & \alpha_{9}\left\{\left[\left(\alpha_{3}+\alpha_{4}\right) \alpha^{3}-6 \alpha^{2}\left(\alpha_{5}-\alpha_{6}\right)(\alpha-1)\right]\left(e^{-\frac{P e_{z}(\alpha-1)}{2}}\right)^{2}(\alpha-1)^{2}-2 \alpha\left(\alpha_{7}+\alpha_{8}\right) e^{P e_{z}}\right. \\
& \left.+\left[\left(\alpha_{3}-\alpha_{4}\right) \alpha^{3}+6 \alpha^{2}\left(\alpha_{5}+\alpha_{6}\right)(\alpha+1)\right]\left(e^{\frac{P e_{z}(\alpha+1)}{2}}\right)^{2}(\alpha+1)^{2}\right\} \mu_{0, H}
\end{aligned}
$$

Similarly, we can find the third and fourth moments. However, due to their lengthy expressions, only plots of these moments are presented in this manuscript.

\section{Numerical Test Problems}

In this section, the analytical solutions presented above are validated by considering several test problems. A second-order accurate finite volume scheme (FVS) is chosen to approximate Eqs. (3)-(12) for verifying the analytical results, see e.g. Javeed et al. (2011). All parameters used in the test problems are given in Table 1. 
Note that, we are working in this study mainly with dimensionless quantities in the model equations. Thus, the general trends identified and discussed below will be valid also for larger column dimensions and additional calculations for a large scale would not bring additional insight.

\section{Rectangular concentration profiles}

In a first series of calculations, influences of boundary conditions, dispersion coefficients and the type of injection are analyzed on the concentration profiles. The sample is either injected through the inner cylindrical core or the outer annular ring. In the calculations described here, the radius of inner cylindrical core $\tilde{r}$ is chosen in such a manner that the inner and outer annular zones have the same areas. Thus, for a column of radius $R=0.2$ the inner zone radius comes out to be $\tilde{r}=0.1414$ (or $\tilde{\rho}=0.707$ ).

A comparison between the analytical solutions for the two types of axial inlet boundary conditions (Dirichlet, Eq. (6), vs. Danckwerts, Eq. (10)) is given in Figure 2 at $x=$ 1 for the case that the sample is injected at $x=0$ through the inner cylindrical core. For the considered selected transport coefficients, $D_{r}=0.03 \mathrm{~cm}^{2} / \min \left(\right.$ or $P e_{r}=0.5$ ) and $D_{z}=0.3 \mathrm{~cm}^{2} / \mathrm{min}$ (or $P e_{z}=20$ ), the two solutions are rather similar. The radial transport is relatively fast. The sample elutes over the complete cross with no visible radial concentration dependence. The local elution profile in the center $(\rho=0)$ and the averaged concentration profiles (Eq. (45)) are also plotted in Figure 2. Due to the rapid radial transport these profiles are almost identical. For the relatively large axial dispersion coefficient assumed, there is a clear difference between the profiles obtained using the Dirichlet or Danckwerts boundary conditions. The more realistic Danckwerts conditions 
quantify the unavoidable back mixing at the column inlet and predict broader profiles.

Figures 3 compares the results shown for injecting the sample through the inner core (Figure 2) with predictions for injecting the same overall amount through the outer annular ring. Due to the rapid elimination of radial concentration gradients the profiles become identical at $x=1$. The plotted temporal profiles of the averaged concentrations depend just on the boundary conditions used.

Figures 4 and 5 provide a comparison of the analytical solutions for injections through the inner and outer zones for less axial back mixing $\left(D_{z}=0.01 \mathrm{~cm}^{2} / \mathrm{min}\right.$ or $\left.P e_{z}=600\right)$ and slower radial transport $\left(D_{r}=0.001 \mathrm{~cm}^{2} / \mathrm{min}\right.$ or $\left.P e_{r}=15\right)$. Now significant radial transport limitations lead to still visible influence of the injection conditions at the column outlet $(x=1)$. Also the local $(\rho=0)$ and averaged concentration profiles differ significantly at the outlet. However, for the rather high $P e_{z}$-number both Dirichlet and Danckwerts BCs give now the same results. The averaged concentrations of both injection cases agree as depicted in Figure 6 .

In the above examples limited inlet sections were used for the injection. Figure 7 provides a comparison of the derived $2 \mathrm{D}$ solutions with the simpler conventional $1 \mathrm{D}$ solutions (obtained using the model in Qamar et al. (2013)), which is included as a special case assuming $\tilde{r}=R$ (or $\tilde{\rho}=1)$. Then there are no radial concentration gradients introduced into the column at the inlet. Due to the assumed constant velocities also no gradients can form internally and both solutions should overlap. For two different sets of transport coefficients the averaged outlet concentration profiles were found to be indeed almost identical.

The results shown in Figure 8 return to the case that the sample is either injected through 
the inner core or the outer ring using $\tilde{\rho}=0.707$. Presented are radial concentration profiles in the middle of the column $(x=0.5)$ for $\tau=1$ assuming different values of the $P e_{\text {rato }}=\frac{R^{2}}{L^{2}} \frac{D_{z}}{D_{r}}$. Hereby, $D_{z}$ was fixed at $0.3 \mathrm{~cm}^{2} / \mathrm{min}$ and $D_{r}$ was varied in the interval $D_{r}=\left[10^{-3}, 10^{-2}, 10^{-1}\right] \mathrm{cm}^{2} / \mathrm{min}$. Inner (upper plot) and outer (lower plot) injections are compared. It can be seen that the imposed step profiles deteriorate faster for larger values of the radial transport coefficients. The two limiting cases corresponding to conservation or elimination of the injection profiles are clearly visible.

Figure 9 gives the comparison of analytical and numerical solutions obtained by finite volume scheme for both sets of boundary conditions. Good agreements can be seen in all solutions.

Analytical solutions for a small radial dispersion coefficient, $D_{r}=0.0003 \mathrm{~cm}^{2} / \mathrm{min}$, are given in Figures 10 and 11 at different positions of the radial coordinate $\rho$ and at the outlet of the column $x=1$. Both inner and outer zone injections are considered for components 1 and 2 characterized by $a_{1}=1$ and $a_{2}=3$, respectively. Due to linear adsorption isotherm, the model equations of both components are decoupled. Therefore, we performed simulations independently by solving the single component equation for each adsorption coefficient. Moreover, $D_{z}=0.3 \mathrm{~cm}^{2} / \min , v=1.0 \mathrm{~cm} / \mathrm{min}$, and $c_{\mathrm{inj}, 1}=1 \mathrm{~g} / \mathrm{l}=c_{\mathrm{inj}, 2}$. One can observe from the figures that there are considerable variations in the concentrations of both components along the radial coordinate of the column. If the injection is introduced through the inner core the highest concentrations appear at the outlet and at the center of the column. For the first component the relative concentration reaches here a value of roughly 0.12 (Figure 10). The trends are similar for both components. For the conditions 
considered severe radial profiles develop. In case of injecting through the outer zone only very low concentrations are observed in the column center at the outlet (Figure 11). The maximum relative concentration is found at the column wall as roughly 0.1 , thus lower than in the case of the inner injection. The averaged concentration profile, averaged over the radial coordinate, are identical for both types of injection (Figures 10 and 11) as could be expected for the assumptions made, i.e. the constancy of the linear velocity and the linearity of the isotherms.

\section{Moments of the solution profiles}

In this study, plots of moments using the Danckwerts BCs are presented. The Dirichlet BCs produced similar results which are therefore omitted.

Figure 12 displays the local moments $\mu_{i}(\rho)$ (c.f. Eq. (49)) plotted along the radial coordinate of the column. The effect of radial dispersion coefficient on the first second, third and fourth moments can be clearly seen. Here, $D_{z}=0.3 \mathrm{~cm}^{2} / \mathrm{min}$ and $u=1.5 \mathrm{~cm} / \mathrm{min}$ were kept fixed and varied was the ratio $P e_{\text {ratio }}=\frac{R^{2} D_{z}}{L^{2} D_{r}}$ which corresponds to $D_{r}=$ $\left[10^{-3}, 10^{-2}, 10^{-1}\right]$. The plots of this figure show that moments approach to constant values along the radial coordinate for smallest value of $P e_{\text {ratio }}$ or largest $D_{r}$. For the smallest value of $P e_{\text {ratio }}=0.075$, the results correspond to the $1 \mathrm{D}$ results presented in Appendix $\mathrm{A}$ of Qamar et al. (2013). Since the concentration is injected via the inner cylindrical core, all moments do not change close to the column center. The changes clearly occur in the outer section. Although the trends look similar, on inspecting closer the $y$-axis, the magnitudes reveal that higher moment change more significantly with changing the $P e$-ratio. Similar 
trends were also observed in the case of injection through outer zone.

Figure 13 illustrates the effect of axial dispersion coefficient (or $P e_{z}$ ) on the first, second, third and fourth averaged moments (c.f. Eq. (51)). One can see the well-known fact that the first moment, corresponding to the retention time, is not affected by the axial dispersion coefficient. Moreover, all these averaged moments are not affected by the values of $D_{r}$. They correspond to moments in the 1D case presented in Appendix A of Qamar et al. (2013). At $1 / P e_{z}=0.5$ (or $D_{z}=0.3$ ), the results given in Figure 13 agree with the results of Figure 12 for the smallest value of $P e_{\text {ratio }}$.

Figure 14 gives the plots of averaged central moments as functions of flow rate. These averaged moments are obtained using the relations in Eqs. (52)-(55). The concentration was injected though inner zone. It was also found that both inner and outer zones injections produce the same results provided the same areas of injection are used. In all cases good agreements can be observed between analytically and numerically determined moments. Moreover, the results again agree with the those of 1D moments presented in Appendix A of Qamar et al. (2013).

\section{Conclusion}

This article presented the analytical solutions and moment analysis of a two-dimensional chromatographic model in cylindrical geometry. Constant flow rates and linear adsorption isotherms were considered. Two sets of boundary conditions were considered, including injections through inner and outer regions of the column inlet cross section. The finite Hankel transform technique in combination with the Laplace transform method were applied 
to solve the model equations. The developed analytical solutions illustrate the influence and quantify the magnitude of the solute transport in radial direction. A comparison of Dirichlet and Danckwerts boundary conditions verified that the predicted elution profiles differ significantly for large dispersion coefficients. For further analysis of the solute transport behavior, the temporal moments up to the fourth order were derived from the Laplace-transformed solutions. The analytical solutions were compared for validation with numerical solutions using a high resolution flux limiting finite volume scheme. Good agreements were observed in the analytically and numerically determined concentration profiles and moments. Results of different case studies were presented and discussed covering a wide range of mass transfer characteristics. The derived analytical solutions provide useful tools to determine longitudinal and radial dispersion coefficients from moments determined experimentally based on integrating recorded elution profiles.

Work is in progress on the extension of the model to variable flow rates and non-isothermal reactive chromatography.

\section{References}

Antos, D., Kaczmarski, K., Wojciecha, P., Seidel-Morgenstern, A., 2003. Concentration dependence of lumped mass transfer coefficients: Linear versus non-linear chromatography and isocratic versus gradient operation. J. Chromatogr. A 1006, 61-76.

Batu, V., 1989. A generalized two-dimensional analytical solution for hydrodynamic dispersion in bounded media with the first-type boundary condition at the source. Water Resour. Res. 25, 1125-1132. 
Batu V., 1993. A generalized two-dimensional analytical solute transport model in bounded media for flux-type finite multiple sources. Water Resour. Res. 29, 288192.

Carslaw, H.S., Jaeger, J.C., 1953. Operational methods in applied mathematics, Oxford University Press, Oxford.

Carta, G., 1988. Exact analytical solution of a mathematical model for chromatographic operations. Chem. Eng. Sci. 43, 2877-2883.

Chalbi, M., 1987. Heat transfer parameters in fixed bed exchangers. Chem. Eng. J. 34, 89-97.

Chen, J.-S., Liu, Y.-H., Liang, C.-P., Liu, C.-W., Lin, C.-W., 2011. Exact analytical solutions for two-dimensional advectiondispersion equation in cylindrical coordinates subject to third-type inlet boundary conditions. Adv. Water Resour. 34, 365-374.

Chen, J.-S., Liu, Y.-H., Liang, C.-P., Liu, C.-W., Lin, C.-W., 2011. Analytical solutions to two-dimensional advectiondispersion equation in cylindrical coordinates in finite domain subject to first- and third-type inlet boundary conditions. J. Hydrol. 405, 522-531.

Coimbra, M.C., Sereno, C., Rodrigues, A., 2003. A moving finite element method for the solution of two-dimensional time-dependent models. Appl. Num. Math. 44, 449-469.

Crank, J., 1975. The mathematics of diffusion, 2nd ed. Clarendon Press, Oxford.

van Genuchten, M.Th., Alves, W.J., 1982. Analytical solutions of the one-dimensional 
convectivedispersive solute transport equation. US Department of Agriculture, Technical Bulletin No. 1661, 151-300.

Guiochon, G., 2002. Preparative liquid chromatography. J. Chromatogr. A, 965, 129-161.

Guiochon, G., Lin, B., 2003. Modeling for preparative chromatography, Academic Press.

Guiochon, G., Felinger, A., Shirazi, D.G., Katti, A.M., 2006. Fundamentals of preparative and nonlinear chromatography, 2nd ed. ELsevier Academic press, New York.

Javeed, S., Qamar, S., Seidel-Morgenstern, A., Warnecke, G., 2011. Efficient and accurate numerical simulation of nonlinear chromatographic processes. Comput. \& Chem. Eng. $35,2294-2305$.

Javeed, S., Qamar, S., Ashraf, W., Seidel-Morgenstern, A., Warnecke, G., 2013. Analysis and numerical investigation of two dynamic models for liquid chromatography. Chem. Eng. Sci. 90, 17-31.

Kubin, M., 1965. Beitrag zur Theorie der Chromatographie. Collect. Czech. Chem. Commun. 30, 1104-1118.

Kubin, M., 1965. Beitrag zur Theorie der Chromatographie. 11. Einfluss der Diffusion Ausserhalb und der Adsorption Innerhalb des Sorbens-Korns. Collect. Czech. Chem. Commun. 30, 2900-2907.

Kucera, E., 1965. Contribution to the theory of chromatography: Linear non-equilibrium elution chromatography. J. Chromatogr. A 19, 237-248. 
Leij, F.J., Skaggs, T.H., van Genuchten M.Th., 1991. Analytical solution for solute transport in three-dimensional semi-infinite porous media. Water Resour. Res. 27, 271933.

Lenhoff, A.M., 1987. Significance and estimation of chromatographic parameters. J. Chromatogr. A 384, 285-299.

Li, P., Xiu, G., Rodrigues, A.E., 2003. Analytical solutions for breakthrough curves in a fixed bed of shell-core adsorbent", AIChE J. 49, 2974-2979.

Li, P., Xiu, G., Rodrigues, A.E., 2003. Modeling breakthrough and elution curves in a fixed bed of shell-core adsorbent for separation of proteins-exact analytical solution and approximate solutions. Chem. Eng. Sci. 59, 3091-3103.

Li, P., Yu, J., Xiu, G., Rodrigues, A.E., 2011. Perturbation chromatography with inert core adsorbent: Moment solution for two-component nonlinear adsorption isotherm. Chem. Eng. Sci. 66, 4555-4560.

Massabó, M., Cianci, R., Paladino, O., 2006. Some analytical solutions for two-dimensional convectiondispersion equation in cylindrical geometry. Environ. Modell. Softw. 21, 6818.

Massabò, M., Catania, F., Paladino, O., 2011. Exact analytical solutions for twodimensional advectiondispersion equation in cylindrical coordinates subject to third-type inlet boundary condition. Adv. Water Resour. 34, 365374.

Miyabe, K., Guiochon, G., 2000. Influence of the modification conditions of alkyl bonded ligands on the characteristics of reversed-phase liquid chromatography. J. Chromatogr. A $903,1-12$. 
Miyabe, K., Guiochon, G., 2003. Measurement of the parameters of the mass transfer kinetics in high performance liquid chromatography. J. Sep. Sci. 26, 155-173.

Miyabe, K., 2007. Surface diffusion in reversed-phase liquid chromatography using silica gel stationary phases of different C1 and C18 ligand densities. J. Chromatogr. A 1167, 161-170.

Miyabe, K., 2009. Moment analysis of chromatographic behavior in reversed-phase liquid chromatography. J. Sep. Sci. 32, 757-770.

Park, E., Zhan, H., 2001. Analytical solutions of contaminant transport from finite one-, two, three-dimensional sources in a finite-thickness aquifer. J. Contam. Hydrol. 53, 4161.

Qamar, S., Abbasi, J.N., Javeed, S., Shah, M., Khan, F.U., Seidel-Morgenstern, A., 2013. Analytical solutions and moment analysis of chromatographic models for rectangular pulse injections, J. Chromatogr. A 1315, 92-106.

Qamar, S., Abbasi, J.N., Javeed, S., Seidel-Morgenstern, A., 2014. Analytical solutions and moment analysis of general rate model for linear liquid chromatography, Chem. Eng. Sci. 107, 192-205.

Ruthven, D.M., 1984. Principles of adsorption and adsorption processes, WileyInterscience, New York.

Schneider, P., Smith, J.M., 1968. Adsorption rate constants from chromatography. A.I.Ch.E. J. 14, 762-771. 
Sneddon, I.H., 1972. The use of integral transforms, McGraw-Hill, New York.

Suzuki, M., Smith, J.M., 1971. Kinetic studies by chromatography. Chem. Eng. Sci. 26, 221-235.

Suzuki, M., 1973. Notes on determining the moments of the impulse response of the basic transformed equations. J. Chem. Eng. Japan 6, 540-543.

Thiele, A., Falk, T., Tobiska, L., Seidel-Morgenstern, A., 2001. Prediction of elution profiles in annular chromatography. Chem. Eng. Sci. 25, 1089-1101.

Wolff, H.-J., Radeke, K.-H, Gelbin, D., 1980. Heat and mass transfer in packed beds-IV use of weighted moments to determine axial dispersion coefficient. Chem. Eng. Sci. 34, 101-107.

Wolff, H.-J., Radeke, K.-H, Gelbin, D., 1980. Weighted moments and the pore-diffusion model. Chem. Eng. Sci. 35, 1481-1485.

Zhang, X., Qi, X., Zhou, X., Pang, H., 2006. An in situ method to measure the longitudinal and transverse dispersion coefficients of solute transport in soil. J. Hydrol. 328, 6149. 


\begin{tabular}{|c|c|}
\hline \multicolumn{2}{|l|}{ Nomenclature } \\
\hline \multicolumn{2}{|r|}{ linear adsorption constant (Henry constant ) (-) } \\
\hline \multicolumn{2}{|r|}{ concentration $\left(\mathrm{kmol} / \mathrm{m}^{3}\right)$} \\
\hline $\bar{c}$ & Laplace transformed concentration $\left(\mathrm{kmol} / \mathrm{m}^{3}\right)$ \\
\hline$c_{\mathrm{av}}$ & averaged concentration $\left(\mathrm{kmol} / \mathrm{m}^{3}\right)$ \\
\hline$c_{H}$ & Hankel transformed concentration $\left(\mathrm{kmol} / \mathrm{m}^{3}\right)$ \\
\hline$c_{\text {init }}$ & initial concentration $\left(\mathrm{kmol} / \mathrm{m}^{3}\right)$ \\
\hline$c_{\mathrm{inj}}$ & injected concentration $\left(\mathrm{kmol} / \mathrm{m}^{3}\right)$ \\
\hline$D_{r}$ & radial dispersion coefficient $\left(\mathrm{m}^{2} / \mathrm{s}\right)$ \\
\hline$D_{z}$ & axial dispersion coefficient $\left(\mathrm{m}^{2} / \mathrm{s}\right)$ \\
\hline$J_{i}$ & $i$-th order Bessel function $(-)$ \\
\hline$L$ & column length $(m)$ \\
\hline$M_{i, \mathrm{av}}$ & $i$-th non-normalized averaged moment $(-)$ \\
\hline$P e_{r}$ & radial Peclet number $(-)$ \\
\hline$P e_{z}$ & axial Peclet number $(-)$ \\
\hline$R$ & column radius $(m)$ \\
\hline$r$ & radial coordinate $(m)$ \\
\hline$\tilde{r}$ & radius of inner cylindrical core $(m)$ \\
\hline$s$ & Laplace domain parameter $(-)$ \\
\hline$t$ & time coordinate $(s)$ \\
\hline$t_{\text {inj }}$ & time of injection $(s)$ \\
\hline
\end{tabular}




\begin{tabular}{|ll} 
Nomenclature (continued) & \\
$x$ & linear velocity $(m / s)$ \\
$z$ & dimensionless axial coordinate $(-)$ \\
Greek symbols & axial coordinate $(m)$ \\
$\epsilon$ & external porosity \\
$\lambda_{n}$ & final Hankel transform parameter $(-)$ \\
$\mu_{i, \text { av }}$ & $i$-th averaged moment \\
$\mu_{i, \text { av }}$ & $i$-th averaged moment \\
$\mu_{i, H}$ & $i$-th moment in Hankel domain \\
$\mu_{i, \text { av }}^{\prime}$ & $i$-th averaged central moment \\
$\rho$ & dimensionless radial coordinate $(-)$ \\
$\tilde{\rho}$ & dimensionless radius of inner cylindrical core $(-)$ \\
$\tau$ & dimensionless time coordinate $(-)$ \\
$\tau_{\text {inj }}$ & dimensionless time of injection $(-)$ \\
\hline
\end{tabular}


Table 1: Parameters used in the considered test problems.

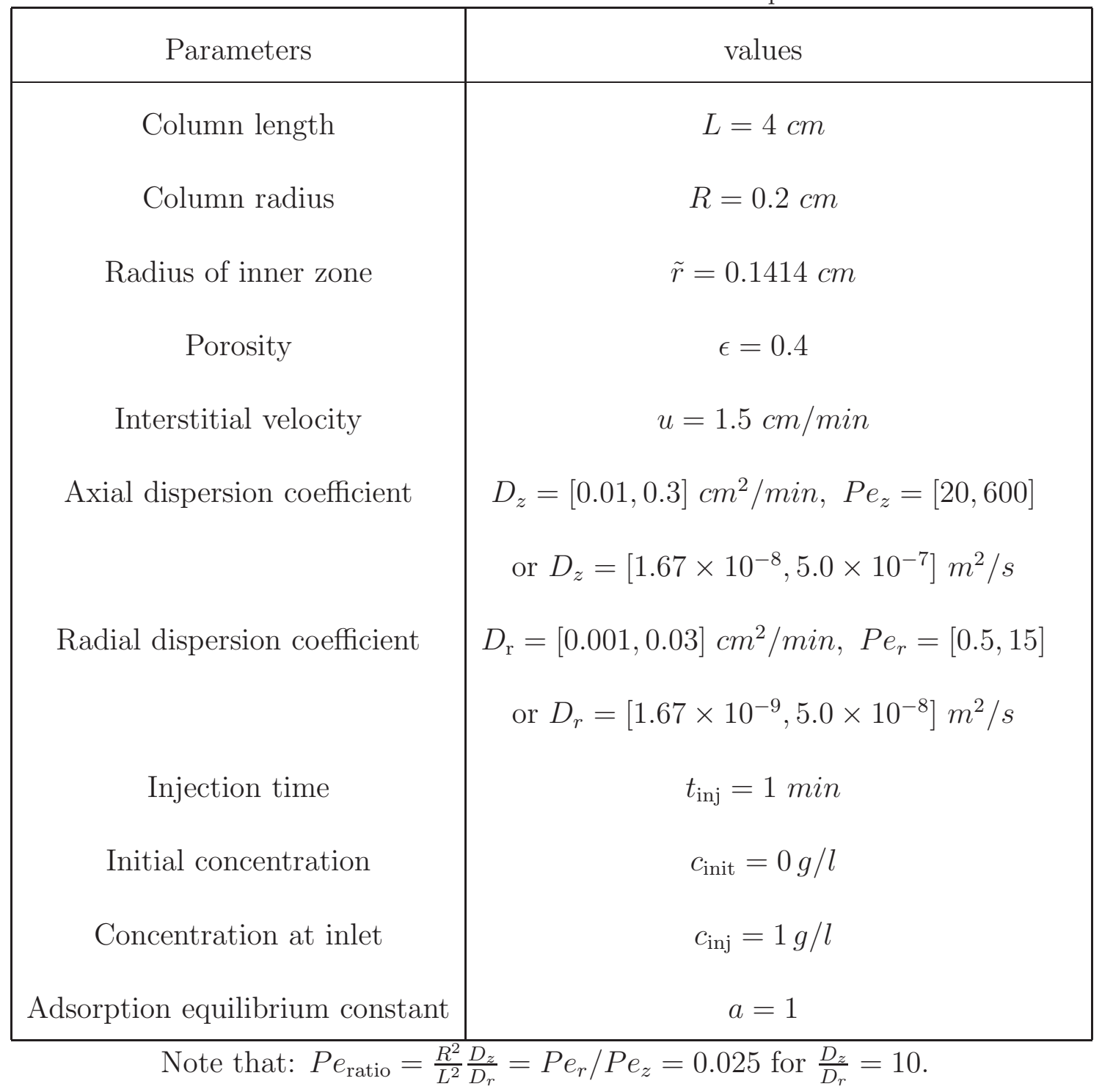




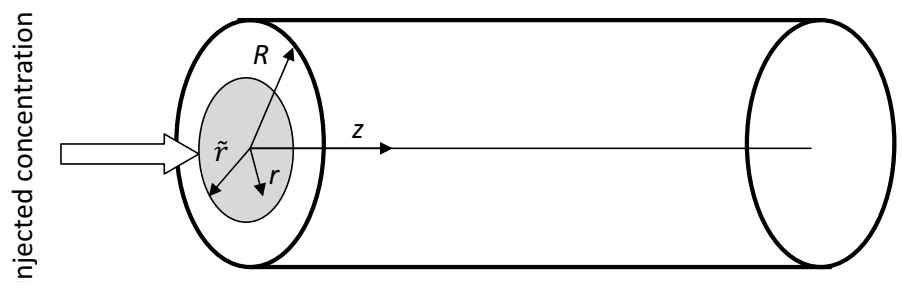

Figure 1: Schematic representation of a chromatographic column of cylindrical geometry. It is assumed that solute can be injected either through inner cylindrical core or through outer annular ring. 

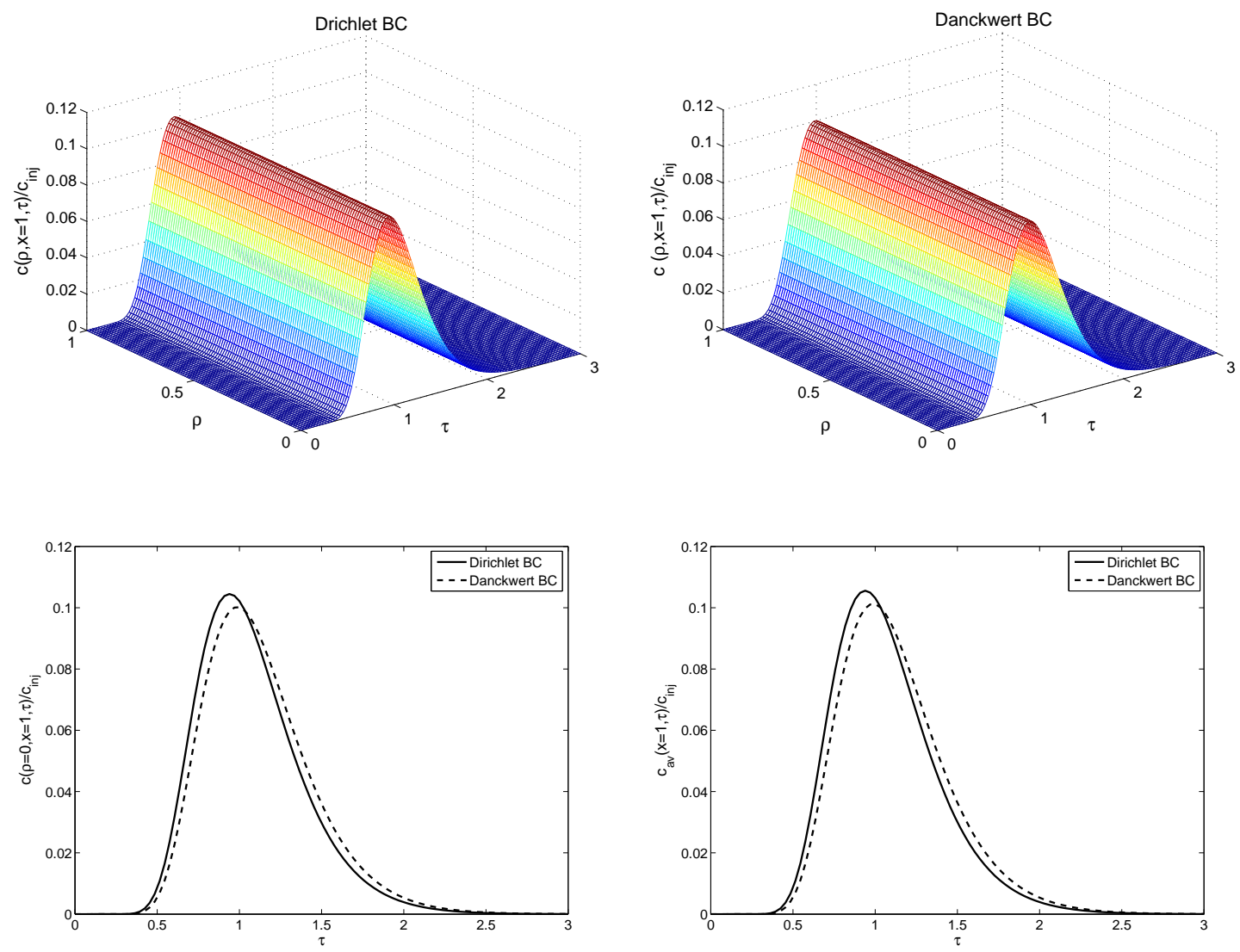

Figure 2: Injection through inner zone of radius $\tilde{\rho}$ : Comparison of solutions for Dirichlet and Danckwerts BCs. Here, $D_{z}=0.3 \mathrm{~cm}^{2} / \min$ (or $P e_{z}=20$ ), $D_{r}=0.03 \mathrm{~cm}^{2} / \min$ (or $P e_{r}=0.5$ ) and other parameters are given in Table 1.

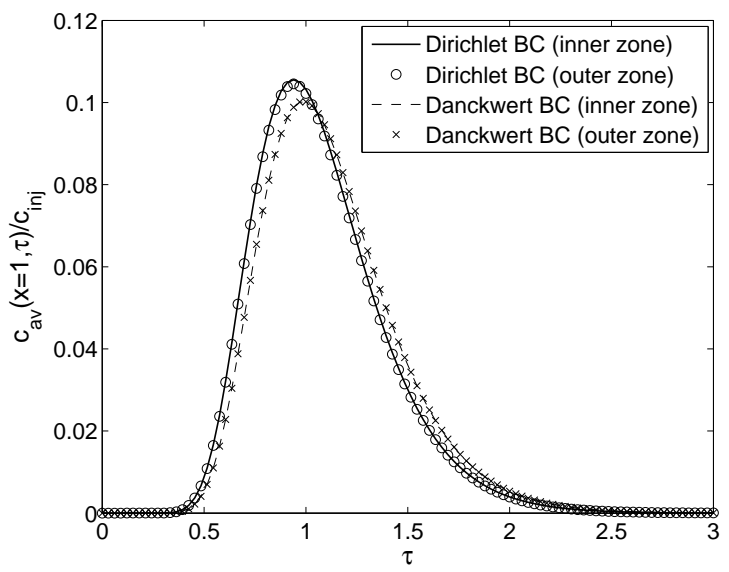

Figure 3: Comparison of averaged concentration profiles obtained from inner and outer zones injections. 35

Here, $D_{z}=0.3 \mathrm{~cm}^{2} / \min$ ( or $P e_{z}=20$ ), $D_{r}=0.03 \mathrm{~cm}^{2} / \min$ (or $P e_{r}=0.5$ ) and other parameters are given in Table 1. 

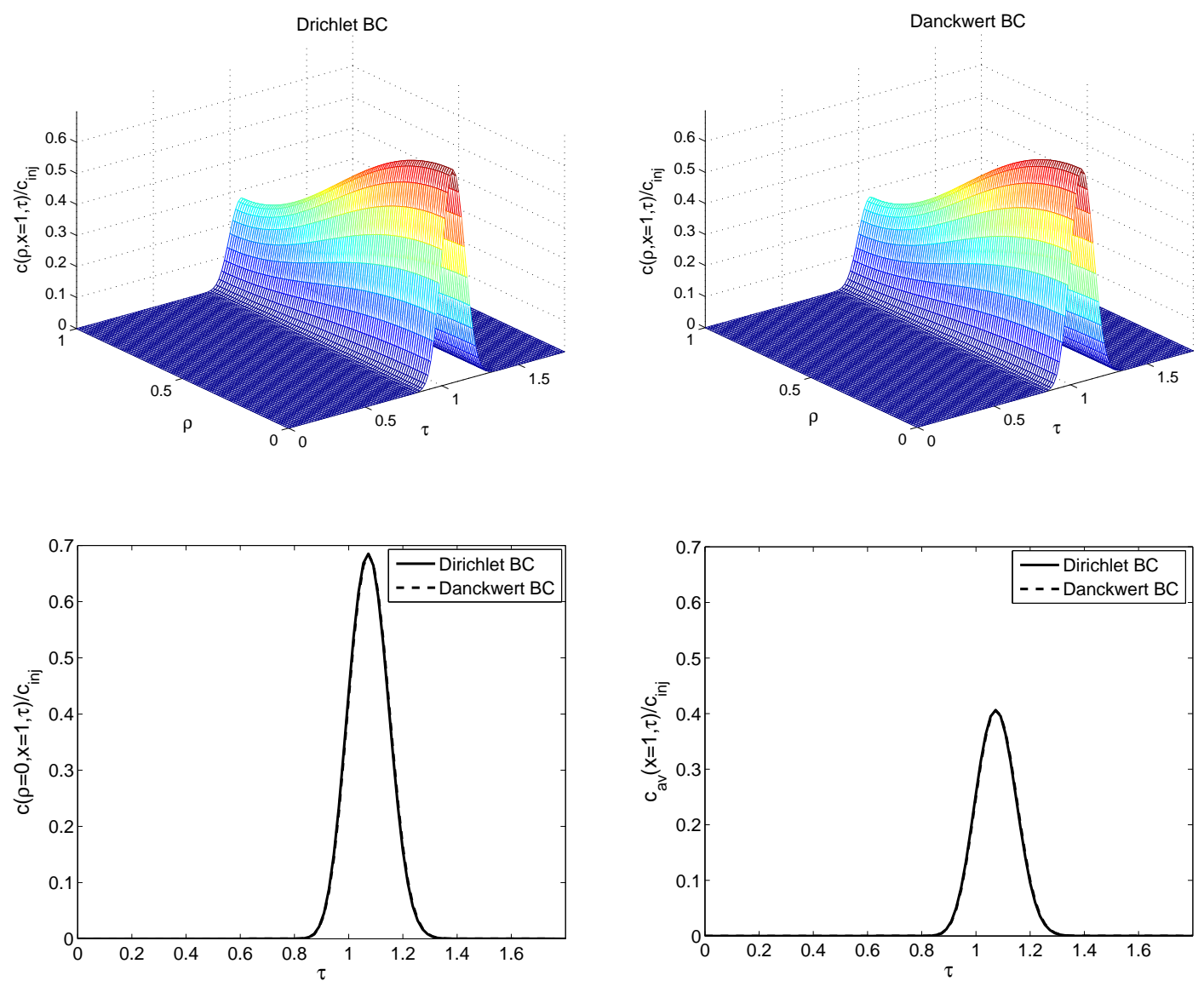

Figure 4: Injection through inner zone of radius $\tilde{\rho}$ : Comparison of solutions for Dirichlet and Danckwerts BCs for $D_{z}=0.01 \mathrm{~cm}^{2} / \min$ (or $P e_{z}=600$ ) and $D_{r}=0.001 \mathrm{~cm}^{2} / \min$ (or $P e_{r}=15$ ). Other parameters are given in Table 1. 

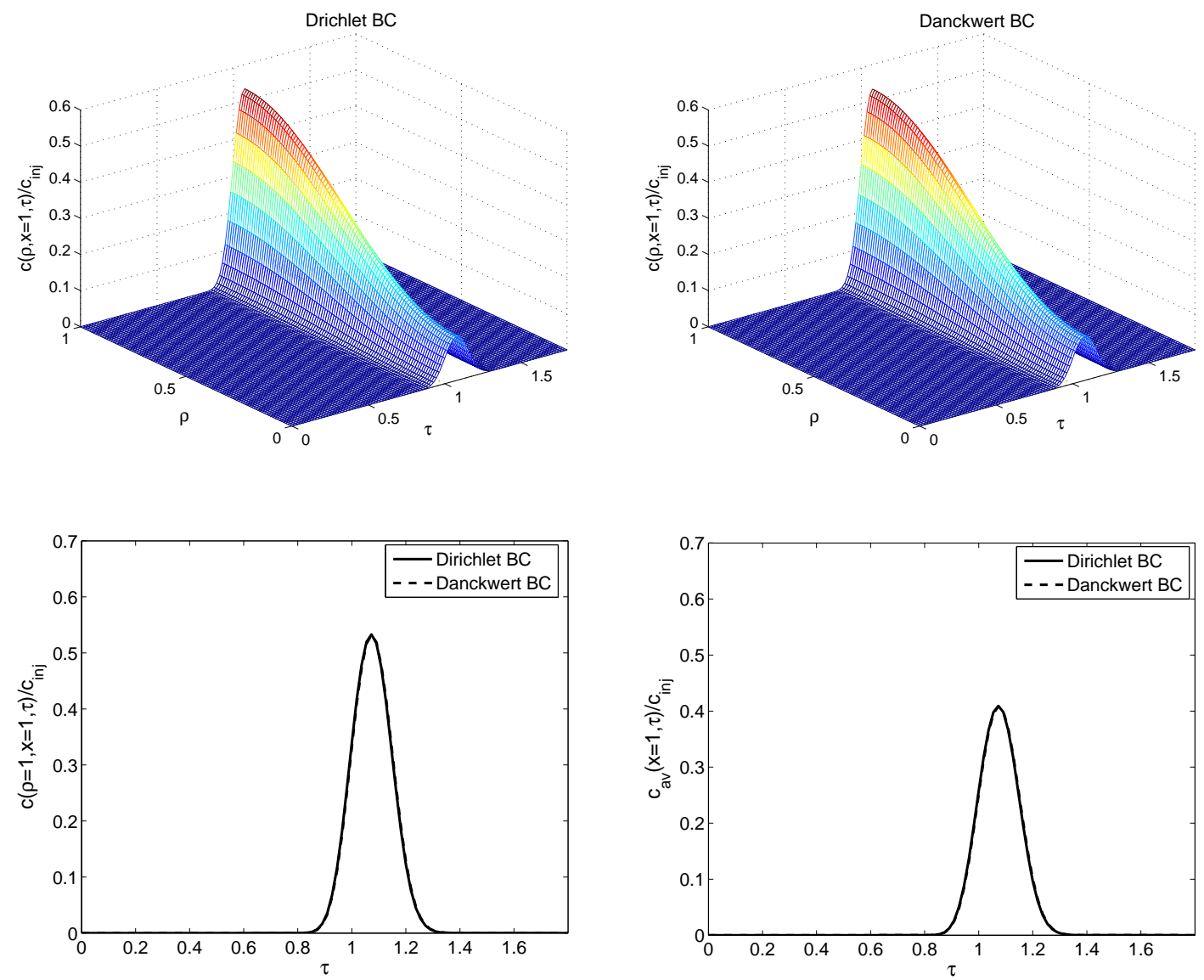

Figure 5: Injection through outer (annular) zone: Comparison of solutions for Dirichlet and Danckwerts BCs for $D_{z}=0.01 \mathrm{~cm}^{2} / \min$ (or $P e_{z}=600$ ) and $D_{r}=0.001 \mathrm{~cm}^{2} / \min$ (or $P e_{r}=15$ ). Other parameters are given in Table 1. 


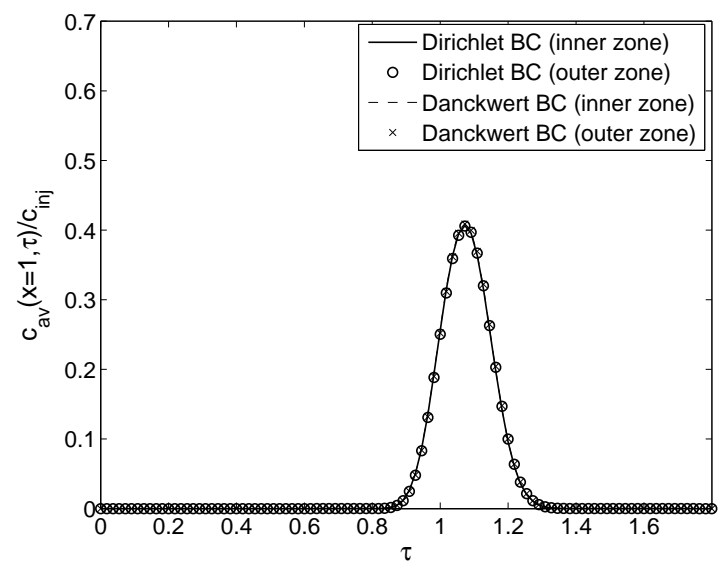

Figure 6: Comparison of averaged profiles obtained from inner and outer zones injections for $D_{z}=$ $0.01 \mathrm{~cm}^{2} / \min \left(\right.$ or $P e_{z}=600$ ) and $D_{r}=0.001 \mathrm{~cm}^{2} / \min \left(\right.$ or $\left.P e_{r}=15\right)$. Other parameters are given in Table 1.
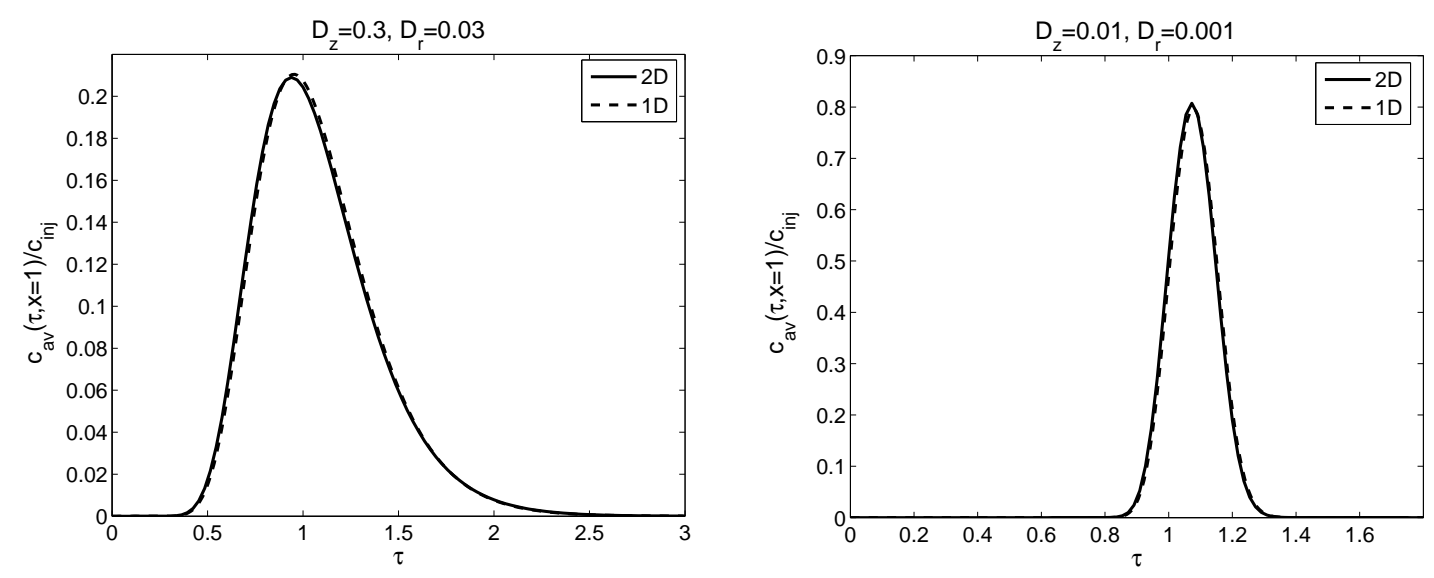

Figure 7: Comparison of $1 \mathrm{D}$ and 2D solutions when $\tilde{\rho}=R$. All other parameters are given in Table 1 . 

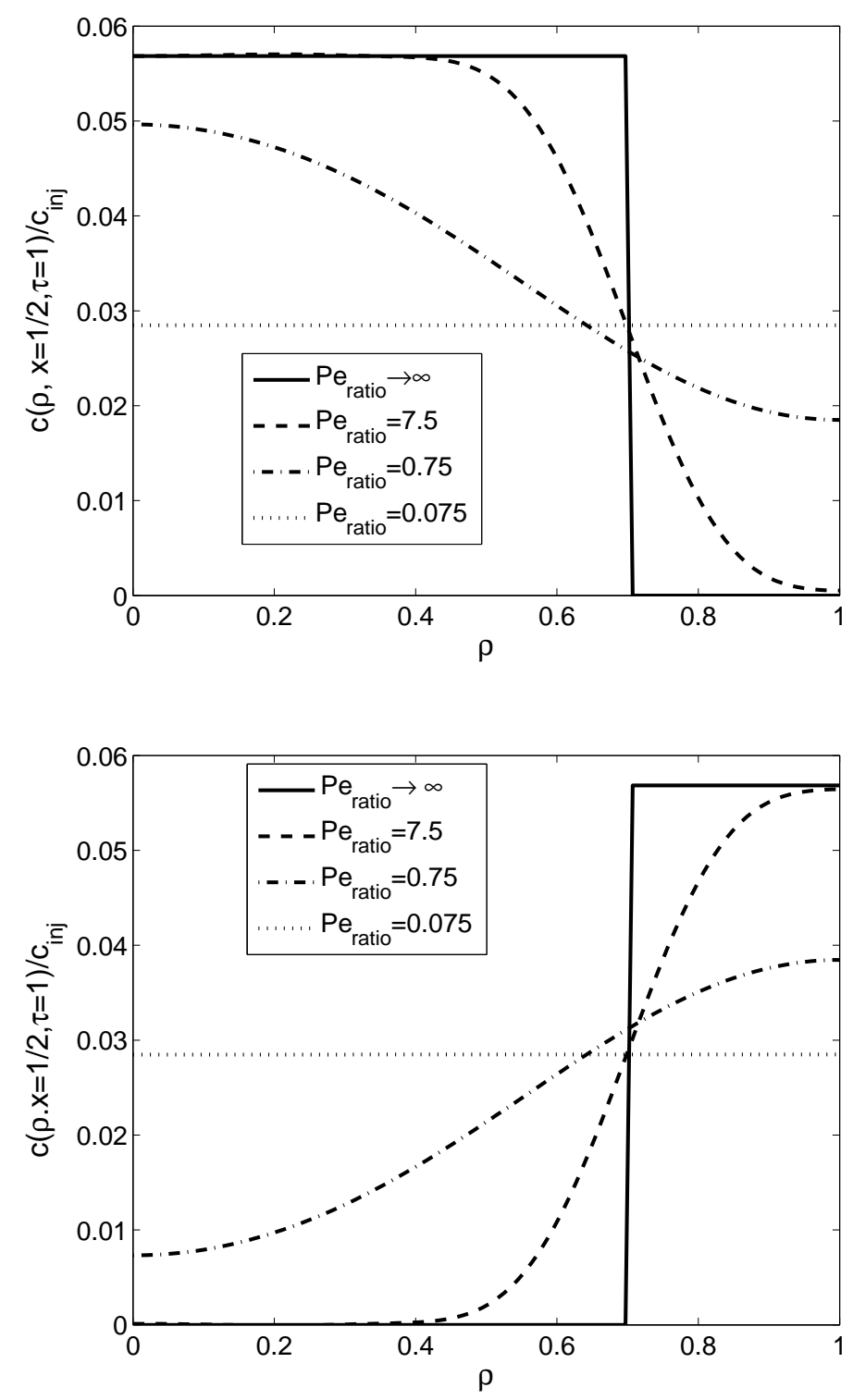

Figure 8: Comparison of solution profiles obtained at $x=1 / 2$ and $\tau=1$ for different values of $D_{r}$ and fixed $D_{z}=0.3 \mathrm{~cm}^{2} / \mathrm{min}$. Here, $P e_{\text {ratio }}=\frac{R^{2}}{L^{2}} \frac{D_{z}}{D_{r}}$. 

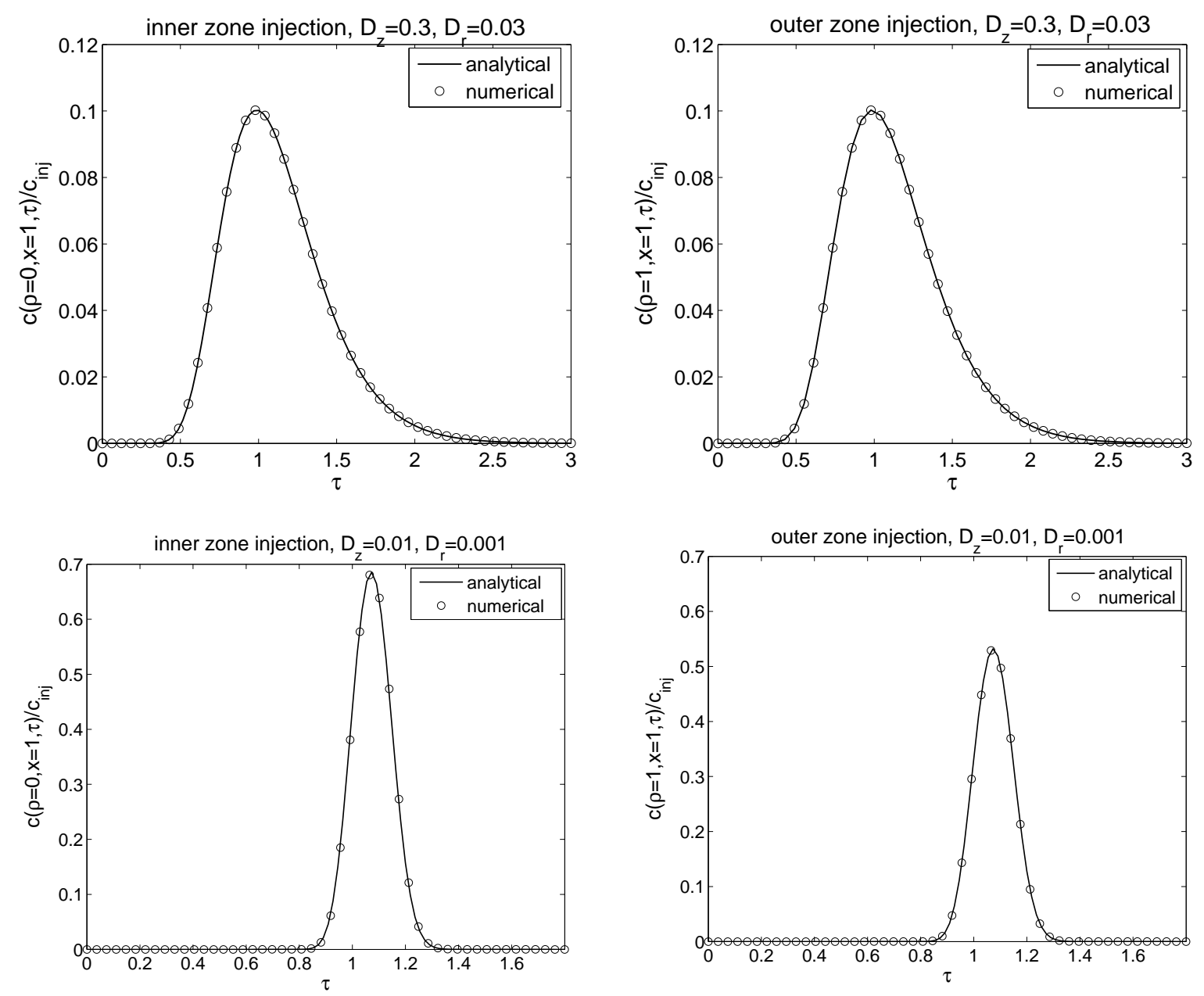

Figure 9: Comparison of analytical and finite volume scheme solutions obtained for different values of dispersion coefficients. Both inner and outer zones injections are considered. 

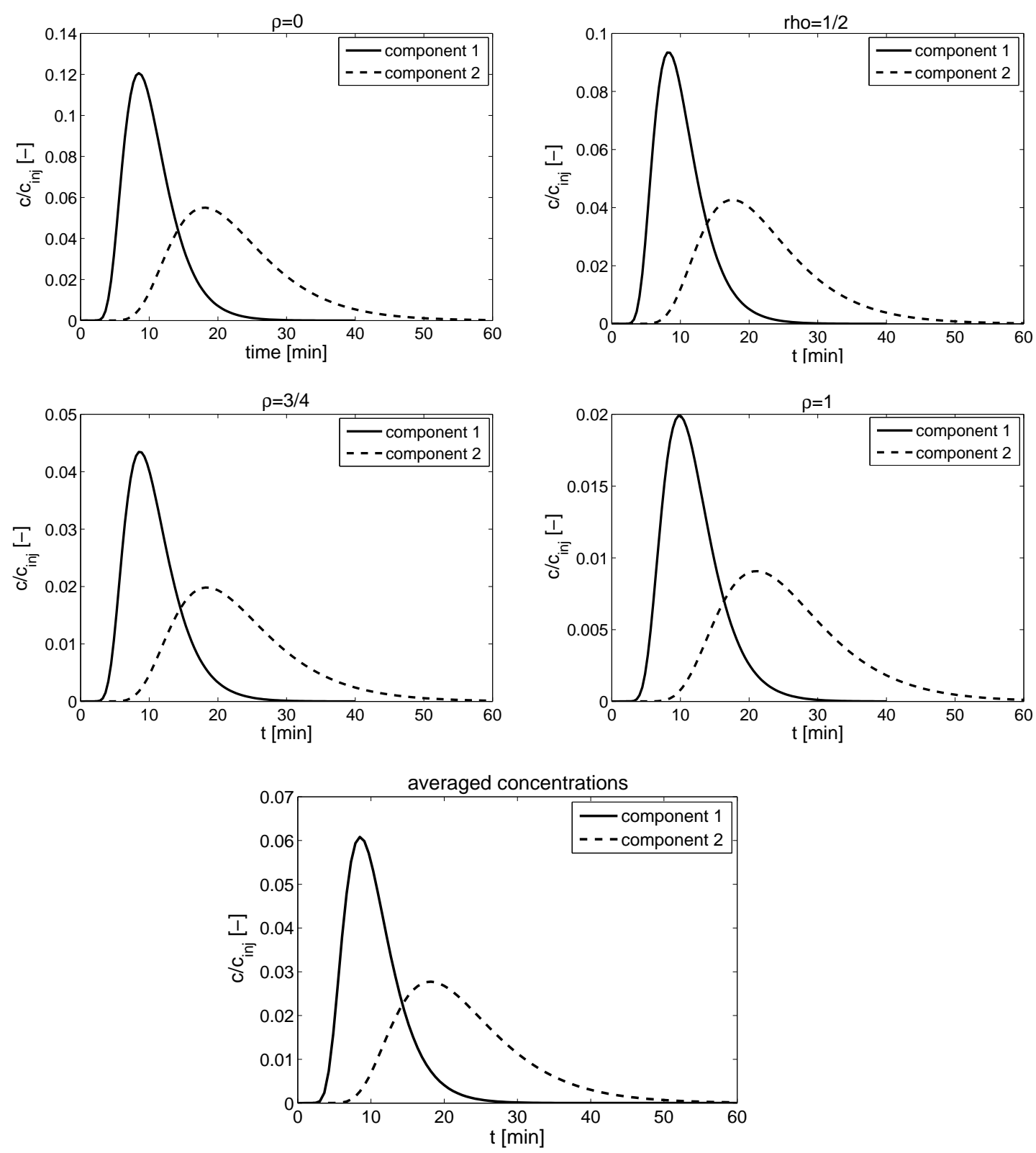

Figure 10: Two-component problem with inner cylindrical core injection: $D_{z}=0.3 \mathrm{~cm}^{2} / \mathrm{min}, D_{r}=$ $0.0003 \mathrm{~cm}^{2} / \min , v=1.0 \mathrm{~cm} / \mathrm{min}, a_{1}=1, a_{2}=3.0$, and all other parameters are given in Table 1 . 

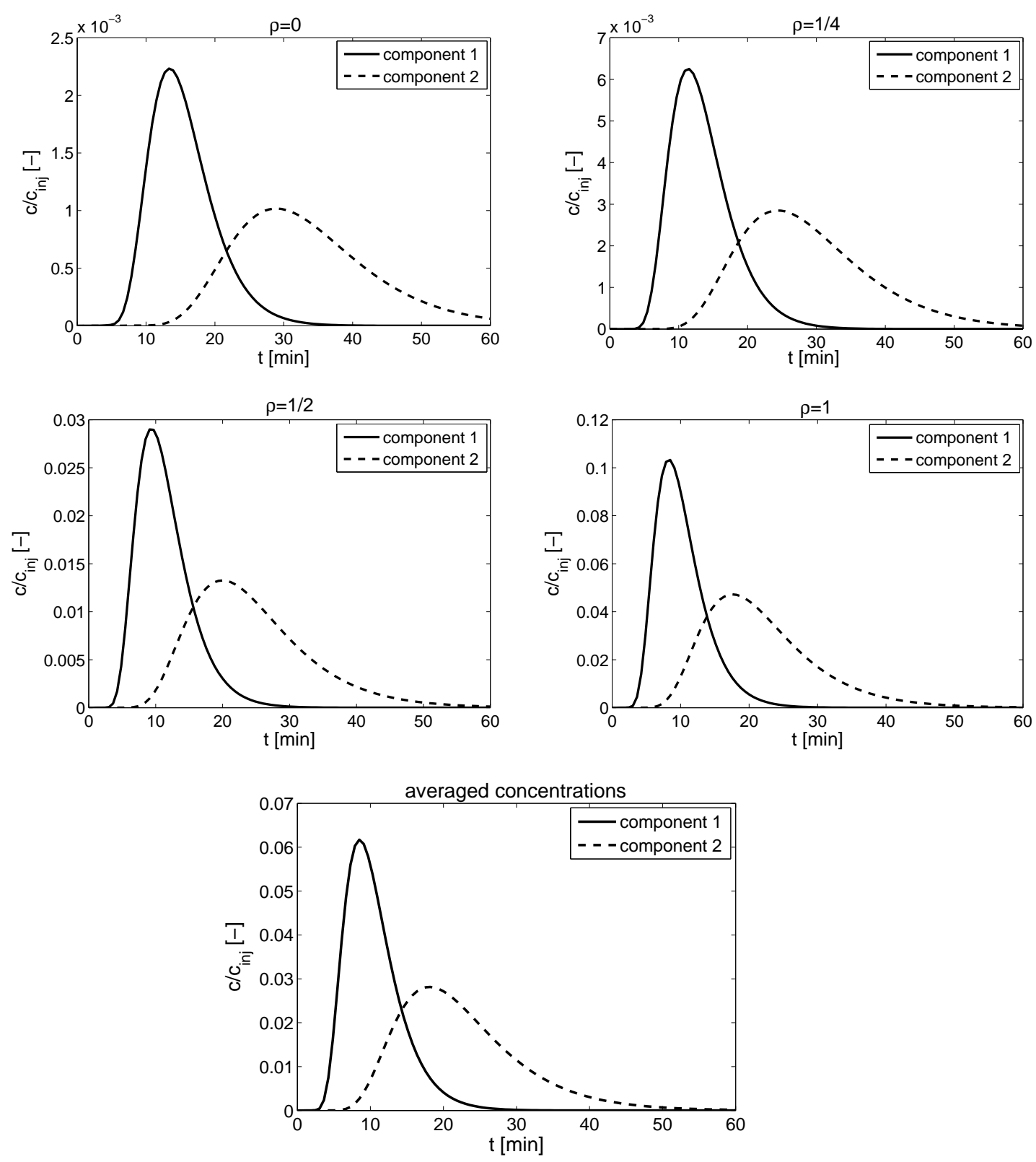

Figure 11: Two-component problem with outer annular zone injection: $D_{z}=0.3 \mathrm{~cm}^{2} / \mathrm{min}, D_{r}=$ $0.0003 \mathrm{~cm}^{2} / \min , v=1.0 \mathrm{~cm} / \mathrm{min}, a_{1}=1, a_{2}=3.0$, and all other parameters are given in Table 1 . 

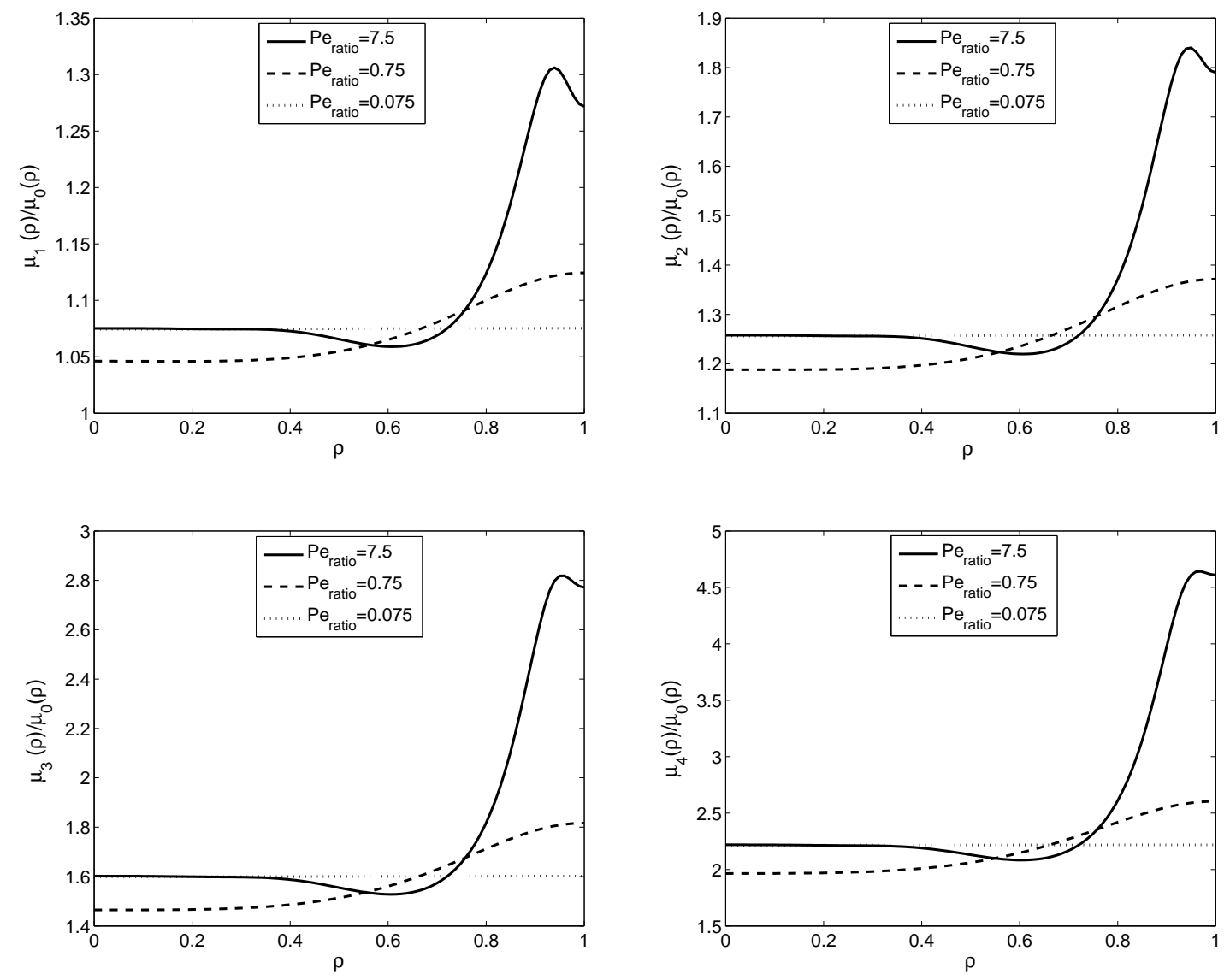

Figure 12: Inner zone injection: Effect of $D_{r}$ on the local moments $\mu_{i}(\rho)$ (c.f. Eq. (49)) for fixed $D_{z}=$ $0.3 \mathrm{~cm}^{2} / \min \left(1 / P e_{z}=0.5\right)$ and $u=1.5 \mathrm{~cm} / \mathrm{min}$. Varied is the ratio $P e_{\text {ratio }}=\frac{R^{2}}{L^{2}} \frac{D_{z}}{D_{r}}$. Other parameters are given in Table 1. For the smallest value of $P e_{\text {ratio }}(=0.075)$, the results correspond to the $1 \mathrm{D}$ case analyzed in Qamar et al. (2013). 

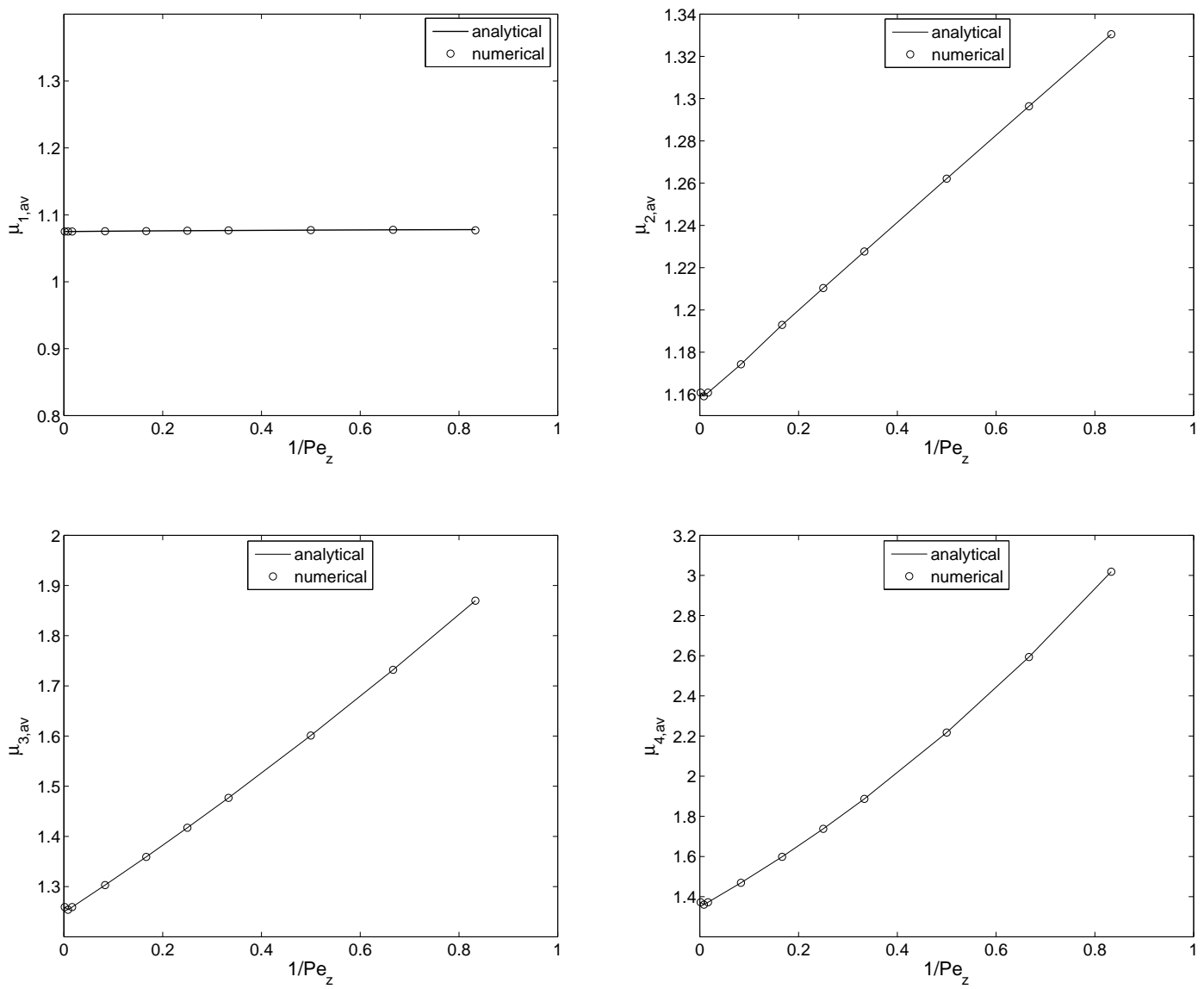

Figure 13: Inner zone injection: Effect of $D_{z}$ (i.e. $P e_{z}$ ) on the averaged moments (c.f. Eq. (51)) for $u=1.5 \mathrm{~cm} / \mathrm{min}$. All other parameters are given in Table 1 . These moments are the same as in the 1D case. 

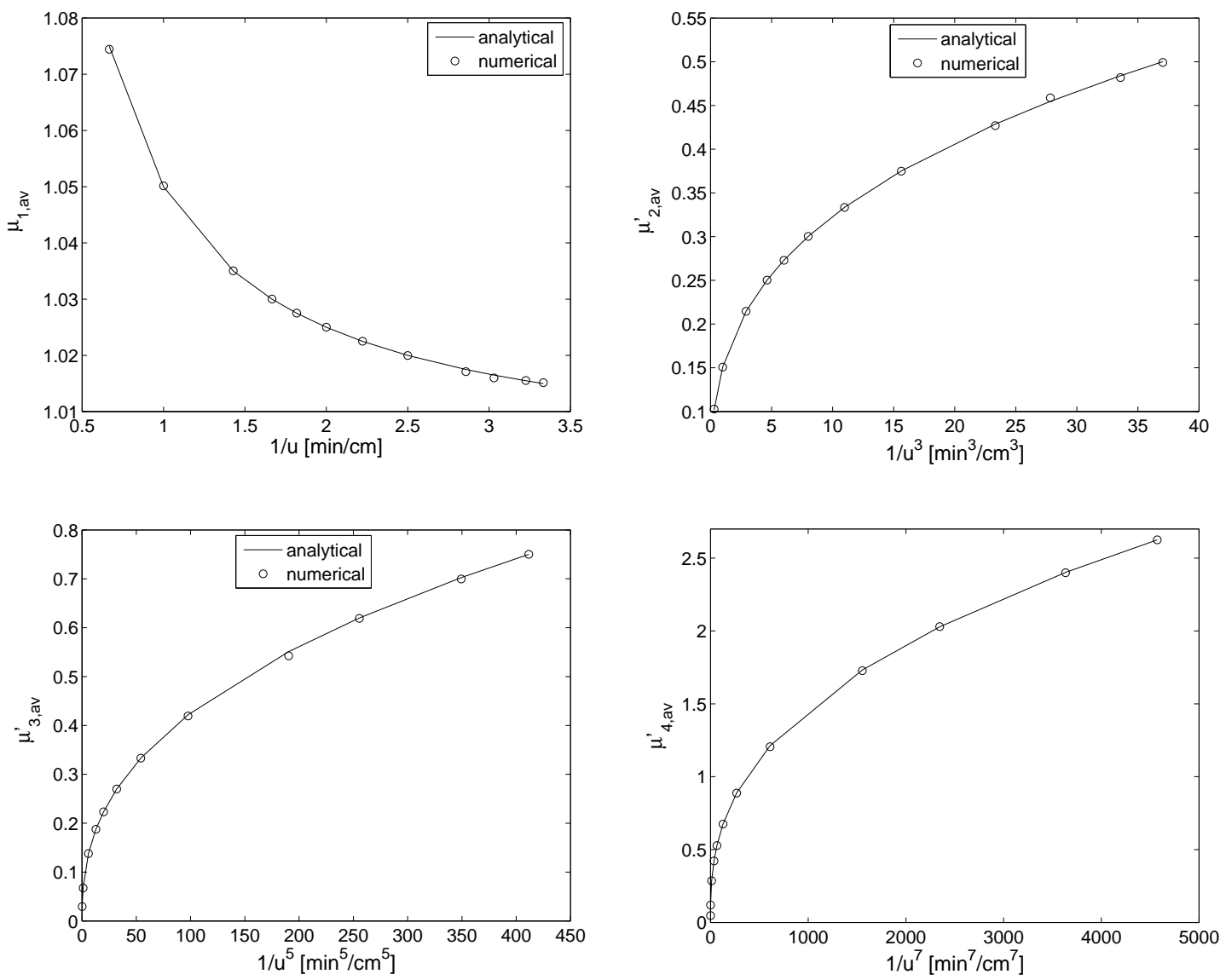

Figure 14: Inner zone injection: Comparison of analytical and numerical averaged central moments (c.f.

Eqs. (52)-(55)) for $D_{z}=0.3 \mathrm{~cm}^{2} / \min \left(1 / P e_{z}=0.5\right)$. All other parameters are given in Table 1 . These moments are the same as in the $1 \mathrm{D}$ case. 